\title{
La relation texte/image dans les notices de jeu de stratégie
}

Text/image relations in strategy game manuals

\section{Mathieu Goux et Pierre Halté}

\section{Q OpenEdition}

1 Journals

\section{Édition électronique}

URL : http://journals.openedition.org/pratiques/8441

DOI : 10.4000/pratiques.8441

ISSN : 2425-2042

Éditeur

Centre de recherche sur les médiations (CREM)

Référence électronique

Mathieu Goux et Pierre Halté, «La relation texte/image dans les notices de jeu de stratégie », Pratiques [En ligne], 185-186 | 2020, mis en ligne le 30 juin 2020, consulté le 15 octobre 2020. URL : http:// journals.openedition.org/pratiques/8441 ; DOI : https://doi.org/10.4000/pratiques.8441

Ce document a été généré automatiquement le 15 octobre 2020

(c) Tous droits réservés 


\title{
La relation texte/image dans les notices de jeu de stratégie
}

Text/image relations in strategy game manuals

\author{
Mathieu Goux et Pierre Halté
}

\section{Introduction}

1 Qu'il soit une activité aux rôles symboliques, sociaux ou ludiques, un jeu doit toujours se dérouler «dans des limites et conditions rigoureuses et constitue une totalité fermée » (Benveniste, 1947, p. 161). En ce sens, et par ce détachement du réel, les participants à une activité de jeu se doivent d'apprendre et d'assimiler une série de contraintes diverses leur permettant de conduire une partie, respecter ses règles et, éventuellement, remporter l'objectif du jeu.

Ces instructions sont généralement délivrées par des notices, par exemple dans le cadre $\mathrm{du}$ jeu de société, sur deux modes distincts: le texte tout d'abord, mais aussi par l'intermédiaire de schémas, tableaux, images et autres supports (icono)graphiques reproduisant ou simplifiant les événements qui prendront place au long d'une partie. Il s'agit donc pour le joueur potentiel d'acquérir une compétence « savoir-jouer » à partir d'un «savoir-lire » complexe, demandant à gérer l'interaction entre ces différentes strates informationnelles.

3 Les relations entre textes et images - souvent analysées par le passé (Hénault, 2008; Macedot-Rouet \& Rouet, 2011; Klinkenberg, 2008, par exemple) - sont des plus élaborées dans le cadre des notices de jeu. Ainsi les concepteurs peuvent-ils illustrer l'univers du jeu dans lequel les gestes conventionnels de la partie prendront place; le texte et l'image interviennent le plus souvent en amont de la partie à proprement parler, lors des phases préparatoires de l'installation du jeu, des flèches et légendes indiquant ainsi le rôle des différentes zones du plateau de jeu (fig. 1). Le texte et l'image peuvent aussi accompagner telle ou telle phase d'une manche, en expliquant comment accomplir une action (fig. 2). Enfin, le texte associé à l'image permet de décrire les 
nombreux éléments présents dans les jeux, leur forme et leur fonction, comme des cartes ou des jetons (fig. 3).

Fig. 1 - Western Trail, p. $4^{1}$

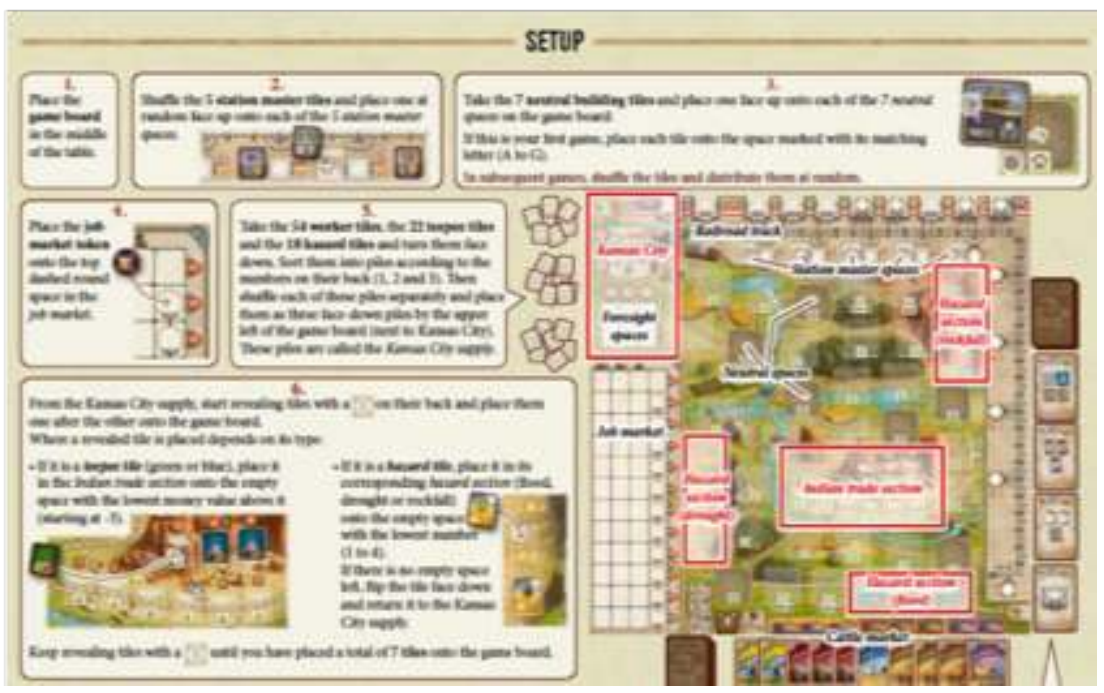

Fig. 2 - Through the Ages, p. 4. Czech Games Edition.

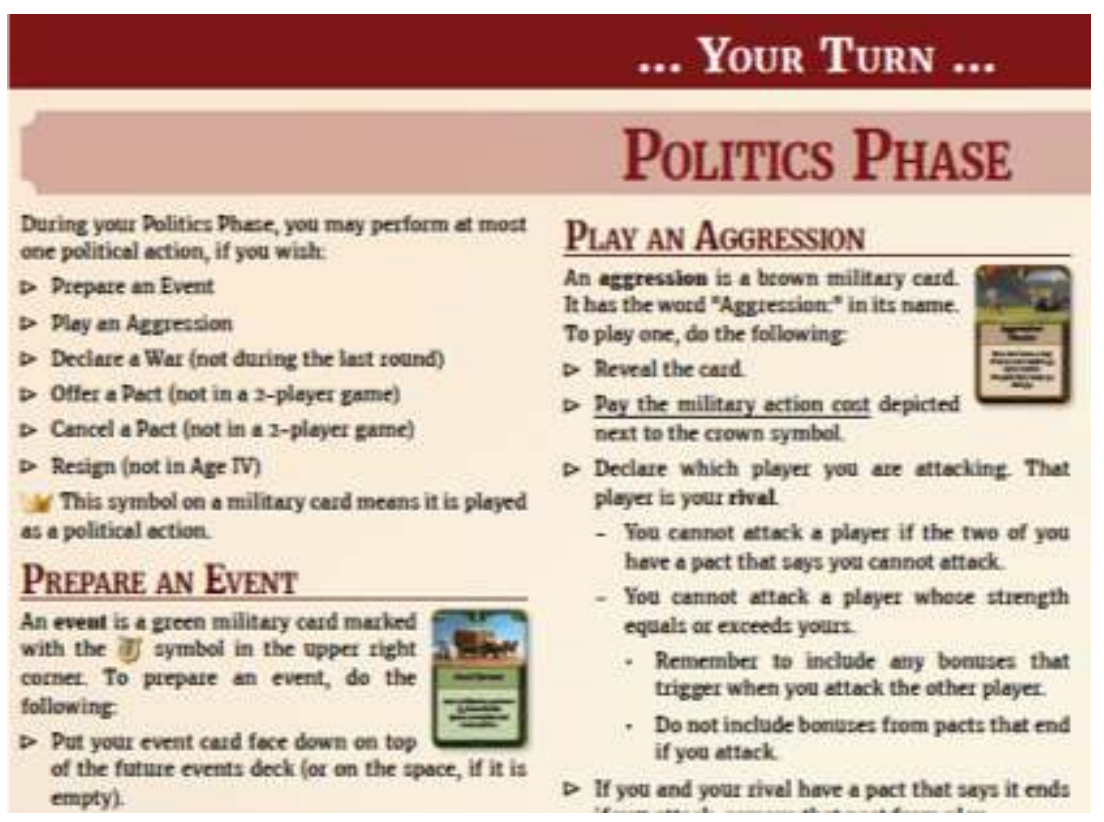


Fig. 3 - Star Wars Rebellion, p. 5. Fantasy Flight Games.

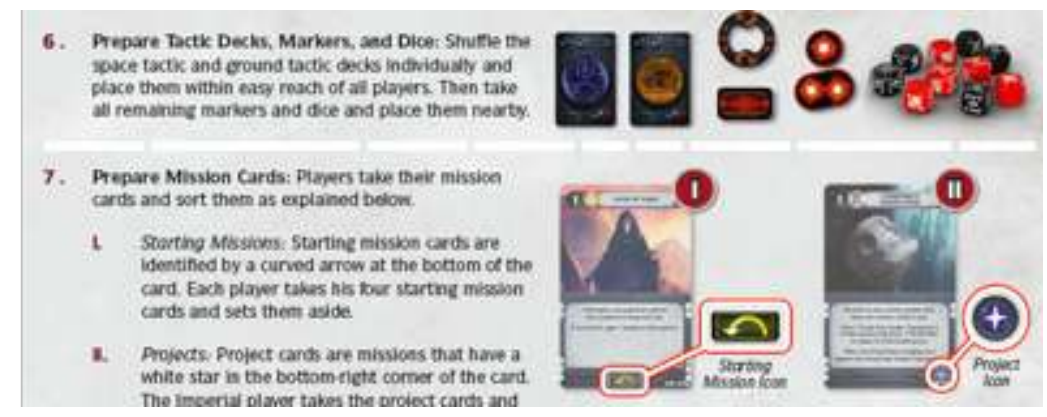

4 Nous proposons dans cet article une étude de ces différentes relations, au sein d'un corpus de dix notices de jeux de société contemporains. Notre perspective ne sera pas de faire un essai de grammaire typologique, à l'instar de ce qu'a pu proposer Klinkenberg (2008), mais de décrire et de circonscrire les fonctions didactiques ${ }^{2}$ de ces différents procédés d'association des textes et des images, ainsi que la séquentialité de ces procédés, dans l'apprentissage des règles d'un jeu par la lecture d'une notice. Nous faisons l'hypothèse que les procédés que nous décrirons ont, certes, un rôle didactique local et spécifique, mais que leur économie (notamment en termes d'agencement et de synergie) au sein de la notice joue, elle aussi, un rôle prépondérant dans l'acquisition des règles par le joueur.

Pour notre travail, nous avons sélectionné les notices de dix jeux de sociétée ${ }^{3}$ Ce sont tous des jeux appartenant au genre de la stratégie, édités entre 2005 et 2018, et classés comme les dix jeux de stratégie les plus populaires sur le site boardgamegeek ${ }^{4}$, référence et base de données incontestée sur internet. Comme la classification générique des jeux de société n'est pas sans difficulté, nous avons repris la typologie proposée par boardgamegeek, sans chercher à la remettre en question. Nous considérons que les jeux de stratégie se caractérisent par une forte implication des joueurs au sein de la partie, qui élaborent à la fois des tactiques à court terme et des stratégies à long terme, prenant en compte leurs atouts et leurs capacités d'action, et qui limitent le recours au hasard ou aux compétences physiques. Les joueurs peuvent être en compétition ou en association les uns avec les autres, et les modalités de victoire divergent selon des paramètres établis en début de partie.

6 Le choix de ce genre s'est imposé à nous pour son exemplarité quant aux phénomènes que nous désirons décrire. Ces jeux se caractérisent par une forte narrativisation, puisque prenant place dans des univers fictifs ou historiques, qui exigent la mise en place d'une diégèse dans laquelle les actions symboliques des joueurs se coloreront d'une interprétation particulière. Ce sont également des jeux d'une grande technicité, demandant à manipuler de nombreux paramètres simultanément ou dans un ordre précis, et d'exploiter de nombreux objets figurant les capacités de jeux tels que des pions, cartes, jetons divers. Ils exigent de séquencer les phases d'apprentissage en différents moments, de la préparation du plateau de jeu au déroulement d'un tour, jusqu'au calcul des points menant à la victoire de tel et tel joueur.

7 Partant, ces caractéristiques exigent de la part des auteurs de la notice du jeu de multiplier les exemples et les moments didactiques, de décrire les tours de jeu et les différents éléments manipulables, le tout en justifiant - ou du moins en incluant - ces phases d'apprentissage au sein d'un univers de fiction. Cette multiplicité nous a permis d'identifier, repérer et classer les différentes façons qu'ont les concepteurs de notice de 
véhiculer des informations à visée didactique. Nous proposons ici un panorama, le plus complet possible, de ces façons de faire, organisé selon des critères qui seront précisés dans la partie 1.1 de l'article. Pour des raisons pratiques de constitution du corpus, nous avons exploité les notices en anglais de ces jeux, l'anglais fût-il ou non la langue d'origine de celles-ci. Dans la mesure où la traduction proposée de ces notices, en français comme en anglais, n'engage ni leur matérialité ni leur agencement, nous n'avons pas pris ce paramètre en considération dans notre étude.

Dans un premier temps, nous décrirons les rapports texte/image observables dans le corpus sous l'angle de leur cadre indexical. Dans un deuxième temps, nous proposerons une typologie de leurs fonctions didactiques. Enfin, dans un troisième temps, nous décrirons la façon dont leur séquentialité détermine les dynamiques d'apprentissage propres au sous-genre de la notice du jeu de société, et potentiellement de la notice de jeu en général.

\section{Les différentes formes des relations texte/image du corpus : marquages indexicaux}

9 La relation entre un texte et une image permet de former des énoncés pluri-codiques dont l'interprétation dépend avant tout de ce que J.-M. Klinkenberg (2008) nomme "cadre indexical ». Cette notion repose sur celle d'index, qui renvoie, en sémiotique peircienne, à un signe ayant une double fonction : d'abord, «focaliser l'attention sur une portion délimitée de l'espace» (ibid., p. 7); ensuite, "donner un statut à cette portion d'espace » (ibid.). En effet, la seule perception visuelle ne suffit pas à associer un texte à une image : cette association nécessite un signe qui l'indique, qui la révèle (c'est, par exemple, pour une peinture dans un musée, le cadre qui l'entoure; ou la case de bande dessinée ; ou encore des formes d'organisation spatiale, plus ou moins discrètes, de la page et des éléments en son sein). Donc, un cadre indexical doit être marqué, cela peut se faire de différentes façons. Avant même que ne prennent place, éventuellement, des calculs sémantiques, liant l'interprétation de l'énoncé verbal à celle de l'énoncé visuel ${ }^{5}$, le lecteur des règles d'un jeu identifie que ces deux éléments sont liés grâce à un dispositif sémiotique parfois complexe, dont l'une des composantes vise à matérialiser le cadre indexical qui regroupe le texte et l'image (voir le cadre rouge, fig. 4). 
Fig. 4 - Twilight Imperium p. 5. Fantasy Flight Games.

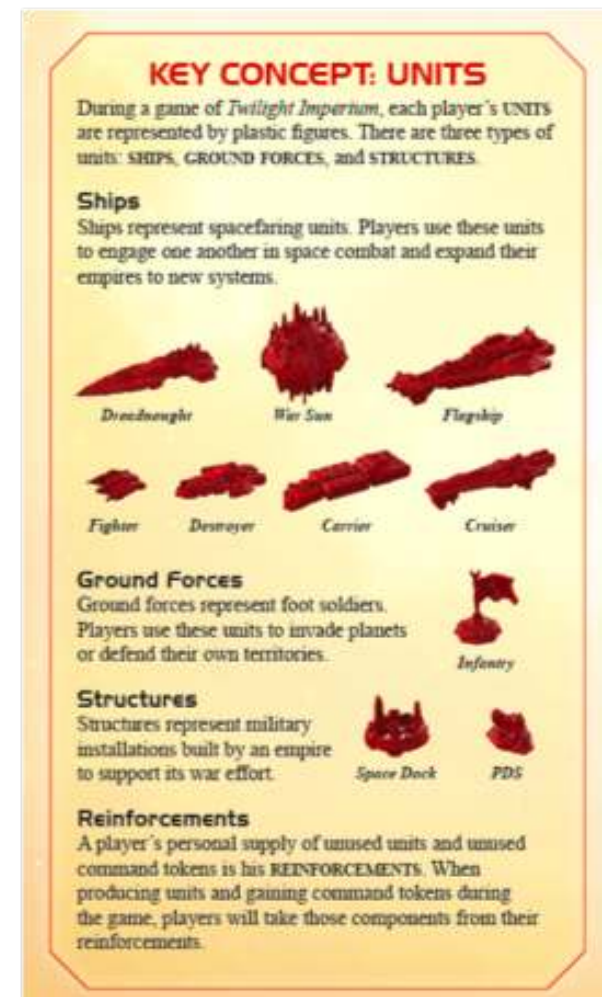

10 Il existe plusieurs niveaux de cadre indexical : pour ce qui concerne la notice de jeu, le cadre indexical le plus large est celui de la page : la présence d'une image et de texte sur une même page nous force à considérer qu'ils sont, d'une manière ou d'une autre, liés, et donc qu'ils forment un système. Au sein de ce cadre très large peuvent coexister une multitude de cadres indexicaux plus étroits et, éventuellement, imbriqués les uns dans les autres.

11 Notre premier niveau de description des rapports texte/image concerne donc ce système de cadres indexicaux - plus ou moins discrètement marqués, mais toujours présents - et l'agencement spatial général du texte et des images en leur sein. Notons au passage que nous ne traiterons pas ici des cas où des pictogrammes s'insèrent dans la chaîne syntaxique, cas qui relève le plus souvent d'un remplacement lexical. Dans ce cas de figure, les auteurs de notice incluent directement au sein de leur texte des pictogrammes en appelant à l'iconographie utilisée dans le jeu, par exemple sur une carte que peut utiliser le joueur (fig. 5). 


\section{Prepare an Event \\ An event is a green military card marked with the in' symbol in the upper right corner. To prepare an event, do the following:}

12 Ce pictogramme, en association, ou non, d'une entrée linguistique, est utilisé à la façon d'un mot ou d'un logotype, dans la mesure où il est parfaitement intégré à la syntaxe de phrase : il prend la place et la position syntaxique d'un «mot » de langue écrite. Dans la mesure où les effets de sens que ce pictogramme produit sont fondamentalement distincts des autres types d'usage de signes iconiques, nous ne les aborderons pas davantage dans cet article ${ }^{6}$.

13 En dehors de ce cas de figure un peu particulier, nous avons identifié trois grandes catégories d'organisation spatiale créant une relation texte/image particulière :

- Séparation entre le texte et l'image ;

- Relation texte/image marquée uniquement par le cadre indexical ;

- Relation texte/image renforcée par d'autres éléments.

\subsection{Séparation entre le texte et l'image}

Le premier cas de figure est celui dans lequel, en dehors de la page de la notice ellemême, aucun cadre indexical ne vient apparemment lier le texte et l'image (fig. 6). Ces images peuvent être positionnées à n'importe quel endroit de la page. Dans les notices de Twilight Imperium et de Star Wars Rebellion, ou encore de Gaia Project, elles sont systématiquement positionnées en bas (indifféremment à droite ou à gauche) des pages. C'est aussi le cas dans la notice de Great Western Trail (p. 15). On trouve dans la notice de Twilight Struggle divers positionnements : en haut à droite, ou en bas à gauche de la colonne de texte. Ici, nous pouvons faire l'hypothèse qu'il s'agit simplement de profiter des espaces vides, dans des sections données des règles du jeu, pour poser des images dont la fonction est avant tout diégétique.

Parfois, l'image et le texte sont même séparés par deux cadres indexicaux bien distincts, comme c'est le cas à la page 12 de la notice de Brass: Birmingham (fig. 7), ou à la première page de celle de Gaia Project (fig. 8), ce qui invite le lecteur à interpréter les deux énoncés comme n'ayant un lien qu'implicite et ténu. 
Fig. 6 - Twilight Imperium, p. 11. Fantasy Flight Games.

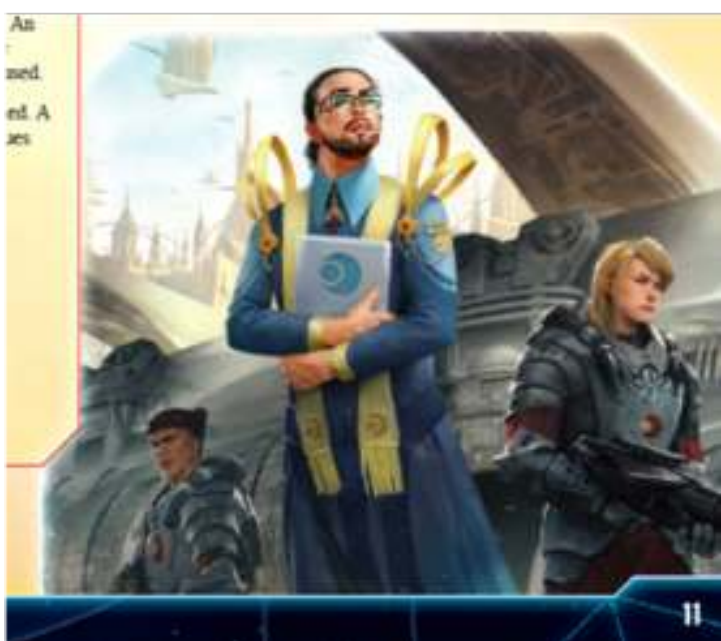

Fig. 7 - Brass: Birmingham, p. 12. Roxley.

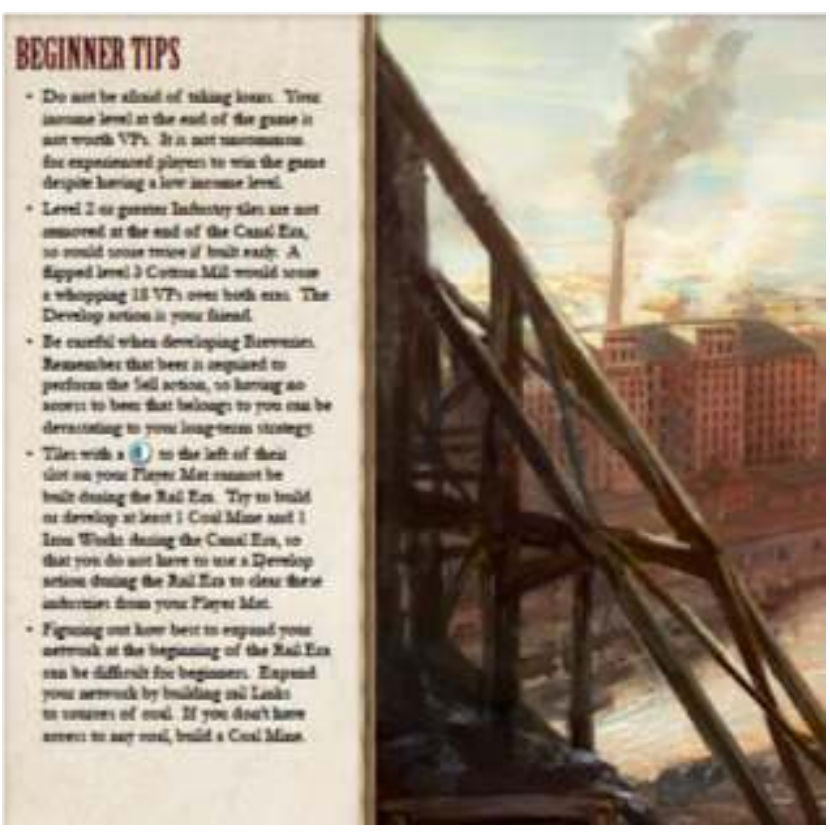


Fig. 8 - Gaia Project, p. 1. Z-Man Games, Inc.

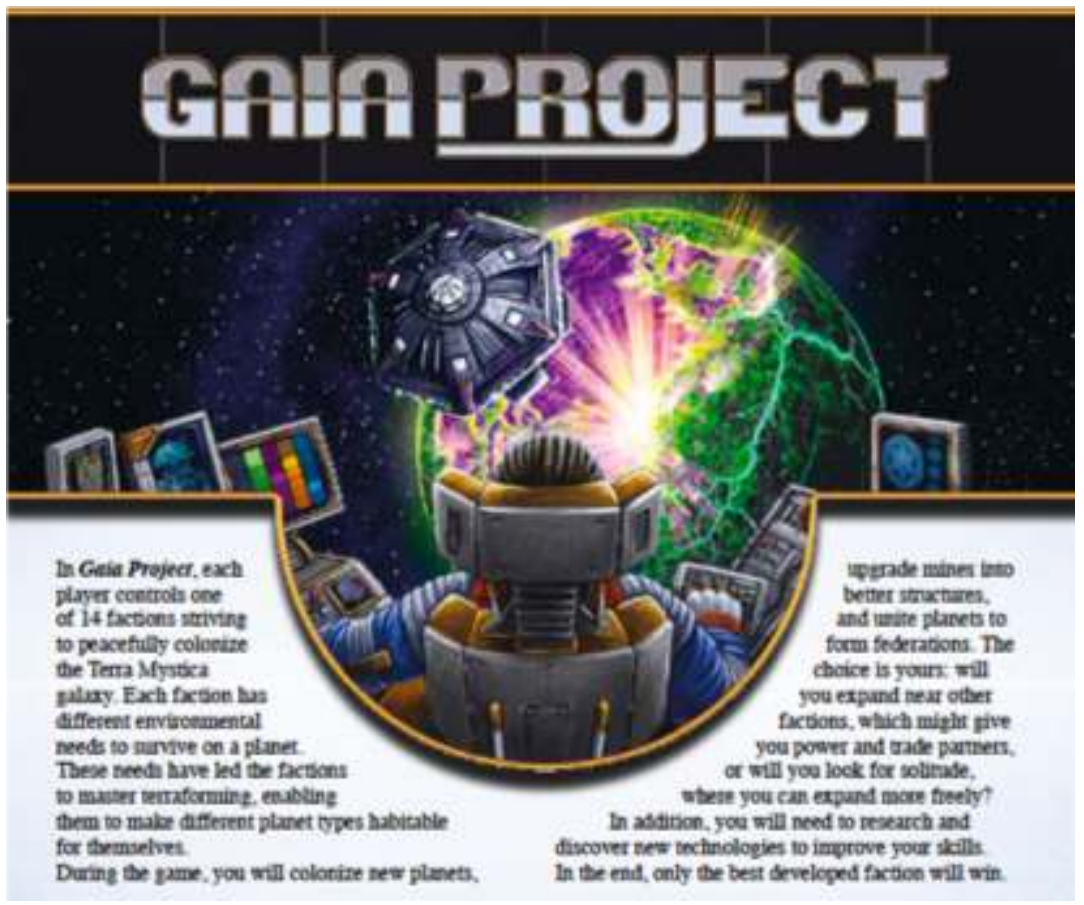

\subsection{Relation texte/image marquée uniquement par le cadre indexical}

Second cas de figure, bien plus fréquent : le texte et l'image apparaissent dans un même cadre indexical, repérable par différents signes, et forçant à les interpréter comme un seul énoncé pluricodique.

17 La façon la plus évidente de construire ce cadre indexical est d'utiliser un marqueur «simple », comme, par exemple, un rectangle dessiné. C'est le cas, par exemple, à la première page de la notice de Brass: Birmingham (fig. 9). 
Fig. 9 - Brass: Birmingham, p. 1. Roxley.

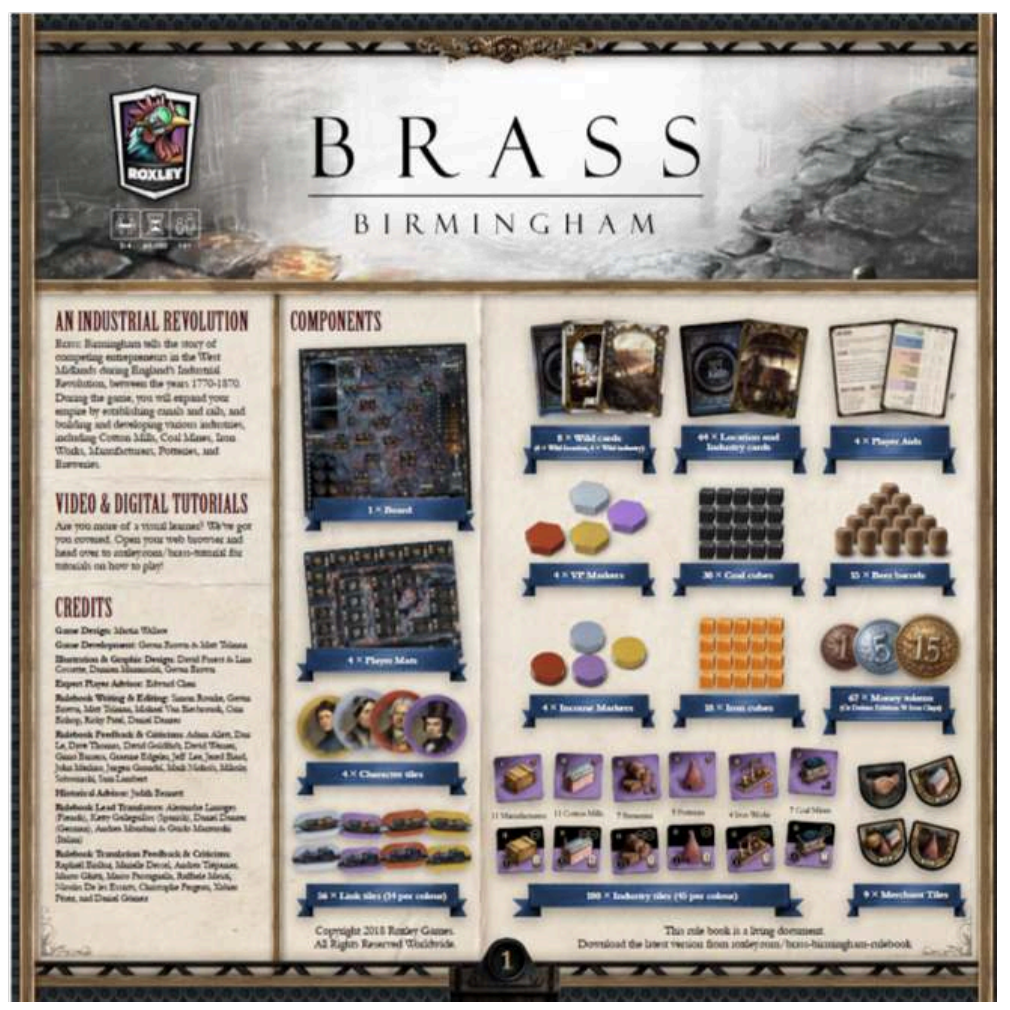
élément de texte, ici par exemple Components («Composants»), qui est interprété comme un titre indexant tous les éléments du cadre dans lequel il apparaît comme des composants de jeu. Il est alors facile pour le lecteur d'identifier tout élément, iconique ou verbal, apparaissant dans ce cadre, comme servant à identifier des éléments de jeu entrant dans la catégorie des «Components ». Très souvent, le cadre explicitement tracé correspond à une charte graphique très précise qui vise à installer un univers fictionnel correspondant à celui du jeu: dans la notice de Brass: Birmingham, les cadres sont marqués par des structures rectangulaires qui ressemblent, dans leur aspect, à du bois ; ils peuvent aussi être signifiés par ce qui ressemble à des pliures dans le papier. La fonction d'indexation des éléments $\mathrm{du}$ cadre est donc doublée d'une fonction diégétique.
Dans la notice de Gaia Project (fig. 10), les cadres indexicaux sont parfois constitués d'un rectangle de couleur différente de celle du fond de la page, avec un effet d'ombrage : 
Fig. 10 - Gaia Project, p. 10. Z-Man Games, Inc.

\section{Phase I: Income}

At the beginning of each round (including the first), income allows each player to gain resources. Income is represented by the icon to the right.

Your faction board, your round booster, your tech tiles, and your current level in each research area on the research board can all allow you to gain income.
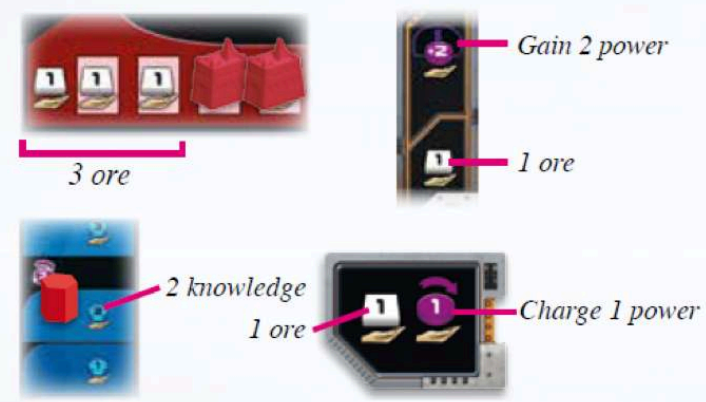

Track your ore, knowledge, and credits using the corresponding tokens on the resource track of your faction board (as you gain or lose a resource, move the token to the number you have remaining). The track ends at 15 , so you cannot have more than 15 ore, 15 knowledge, and 30 credits ( 15 per token).

Parfois, le cadre n'apparait pas explicitement, il n'est donc pas, littéralement, figuré, mais son marquage se fait de façon " implicite " : la spatialisation du contenu de la page oblige le lecteur à considérer certaines associations de texte et d'image comme un seul énoncé pluricodique, et c'est cette spatialisation qui fait office de marquage. C'est le cas par exemple dans la figure 11 : 
Fig. 11 - Star Wars Rebellion, p. 12. Fantasy Flight Games.

\section{THE REBEL BASE}

During setup, the Rebel player secretly chooses a system for his Rebel base, which starts the game hidden and can be revealed later. This section explains all rules for finding, revealing, and moving to and from the Rebel base.

While the Rebel base is hidden, the Rebel player can deploy units in the "Rebel Base" space of the game board without revealing the system in which his base is located. When an effect refers to the "Rebel Base" space, it is referring to this space of the game board and not the system where the Rebel base is hidden.

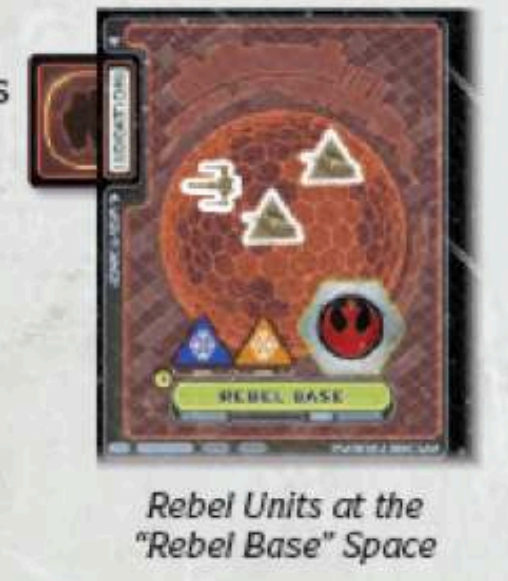

21 On y identifie sans peine ce qui constitue le corps du texte, et, à sa droite, se trouvent des associations d'images et de texte, devant manifestement être interprétées comme un tout cohérent et cohésif, suivant les règles et les conventions habituelles de la légende : l'image est au-dessus, et le texte en italiques, en dessous, décrivant l'image. L'italique permet de bien détacher le texte sous l'image du texte constituant le corps de la règle. Dans ce cas, il est possible de trouver tous les positionnements possibles dans notre corpus: le texte (en italiques, ou en gras) peut être au-dessus de l'image, en dessous, à sa gauche, à sa droite, ces positionnements pouvant fluctuer au sein d'une même page d'une notice particulière, comme le montre la figure 12 :

Fig. 12 - Great Western Trail, p. 3. Eggertspiele.

\section{- 3 Dutch Belt (green)}

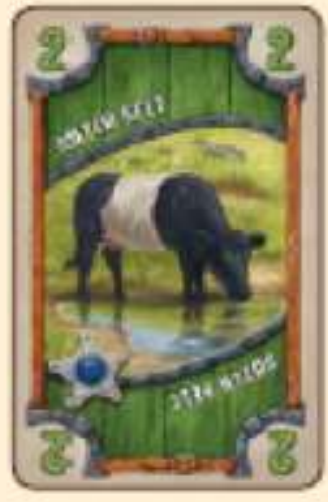

\section{- 35 with a value of 1 Dollar}


22 L'indexation se fait alors par le repérage spatial au sein de la page (le bloc texte/image étant séparé du reste par des blancs), par le contraste qui existe entre le texte servant à accompagner l'image et le texte principal renvoyant à la règle du jeu, et enfin par la proximité entre le texte et l'image.

\subsection{Relation texte/image renforcée par d'autres éléments}

Enfin, dans certains cas, le cadre indexical est, certes, apparent, mais des procédés très variés s'y ajoutent, jouant sur plusieurs systèmes sémiotiques, renforçant et précisant le lien entre les éléments qui y apparaissent. On trouve par exemple, dans la notice de Great Western Trails, un système de phylactères, qui relient des zones de texte à des zones précises d'une image (fig. 13) :

Fig. 13 - Western Trails, p. 4

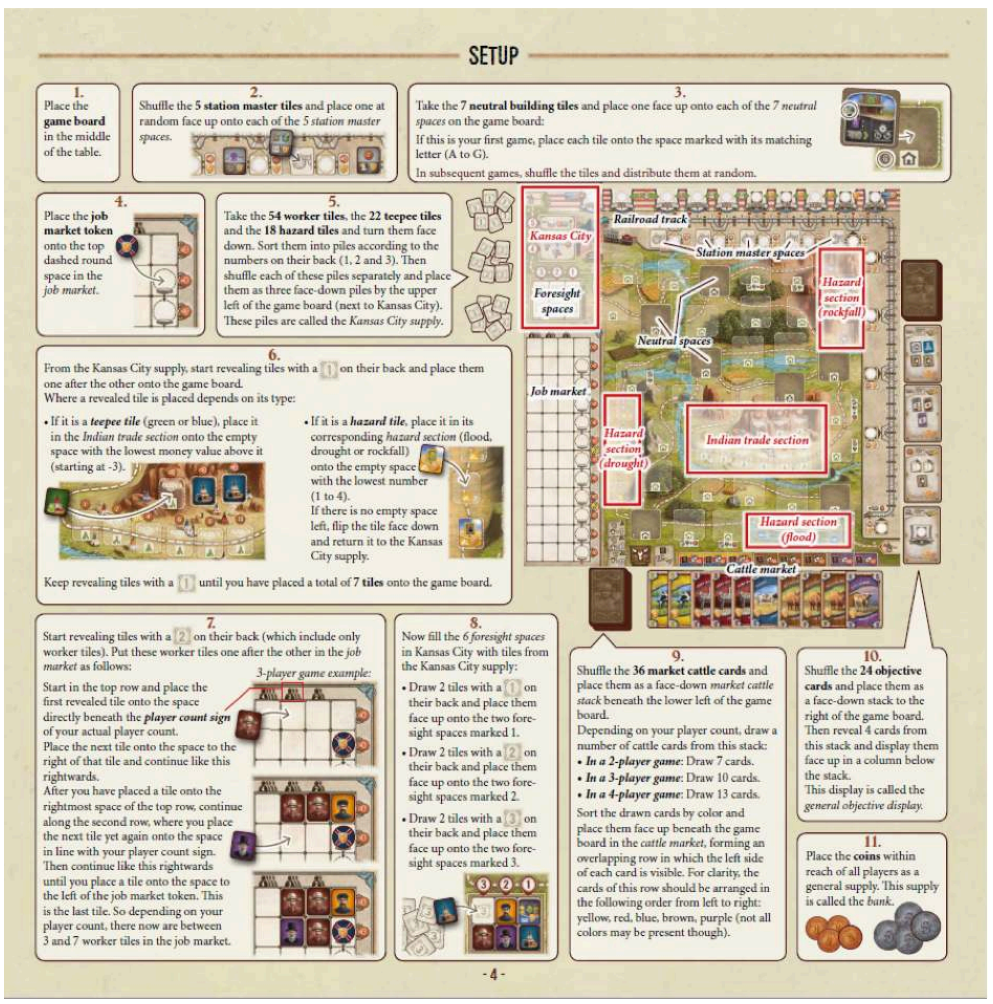

Les flèches constituent aussi un puissant outil d'indexation, et peuvent être utilisées de différentes manières (fig. 14) : 
Fig. 14 - Twilight Imperium, p. 18. Fantasy Flight Games.

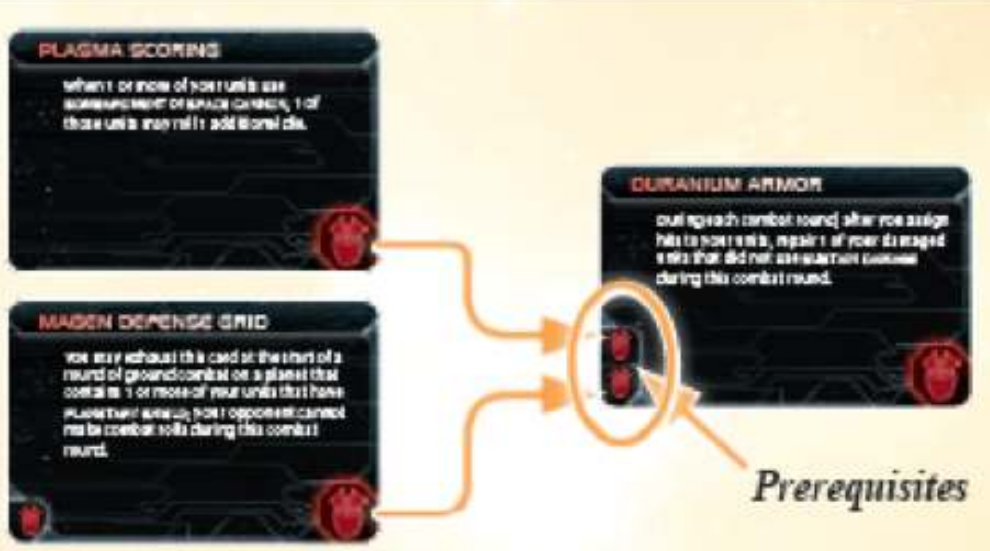

\section{A player needs to own at least two red technologies to research "Duranium Armor" as it has two red prerequisites.}

On trouve des systèmes d'indexation chiffrés, chaque chiffre (ou, parfois, lettre), posé à un endroit de l'image, renvoyant à un texte précédé du même chiffre ou lettre et expliquant la zone de l'image concernée, comme à la page 15 de la notice de Star Wars Rebellion (fig. 15) :

Fig. 15 - Star Wars Rebellion, p. 15. Fantasy Flight Games.

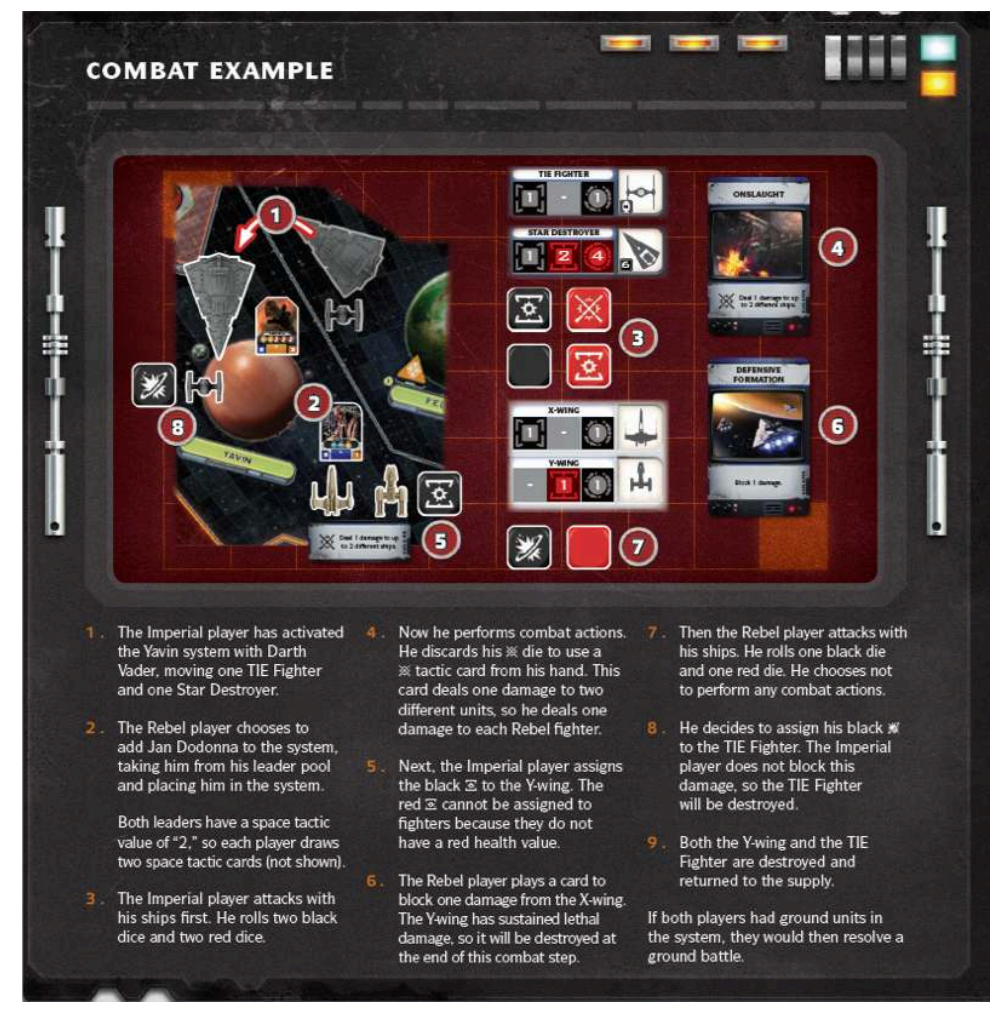

Il est aussi possible de trouver des flèches chiffrées, pour renvoyer à des processus devant être effectués dans un certain ordre (fig. 16) : 
Fig. 16 - Star Wars Rebellion, p. 9. Fantasy Flight Games.

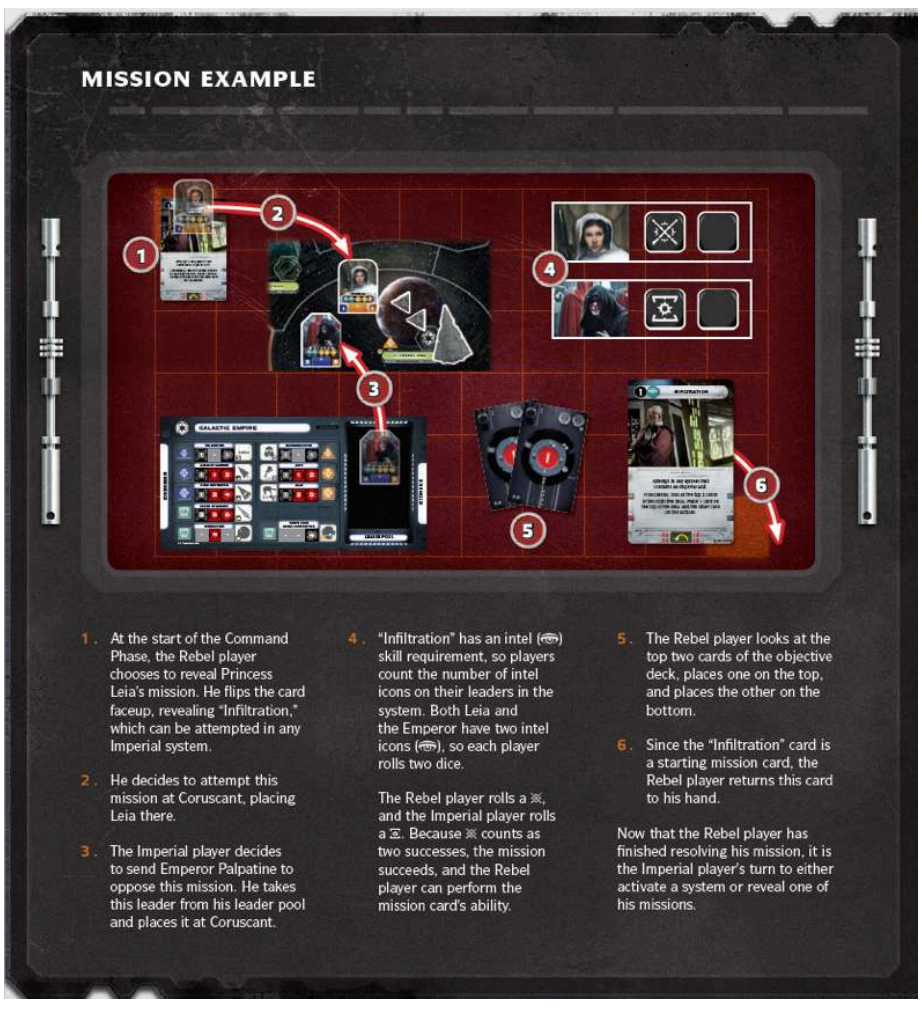

27 Une autre forme d'indexation consiste tout simplement à poser du texte directement sur une zone de l'image, le rapport indexical étant alors établi par contiguïté énonciative. On peut illustrer cela dans la fig. 17 par le texte en noir (« Foresight spaces ", par exemple)et son positionnement qui indique que l'énoncé verbal porte sur la portion de l'énoncé visuel sur lequel il est inscrit, délimitant ainsi une deixis commune à l'énoncé visuel et verbal : 
Fig. 17 - Great Western Trail, p. 4. Eggertspiele.

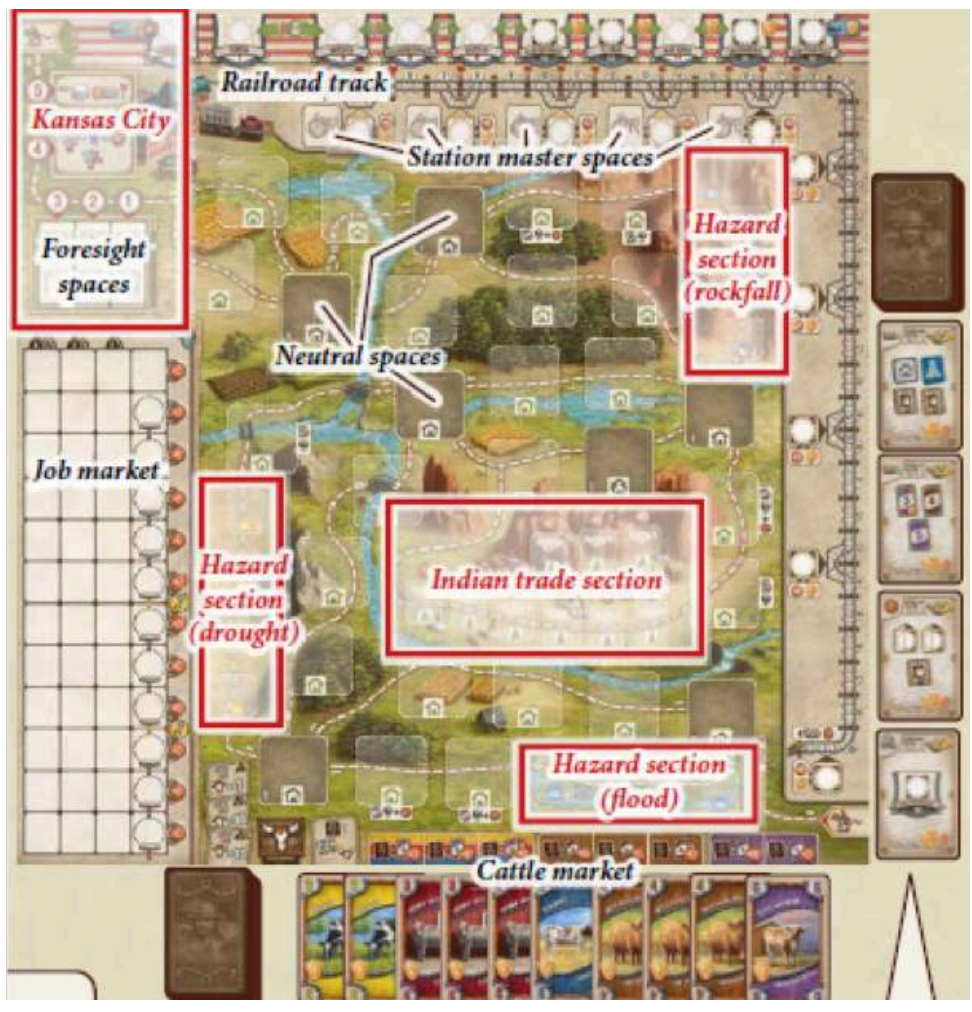

Tous ces procédés peuvent se combiner pour former des systèmes sémiotiques d'une grande complexité. Observons par exemple la figure 18 :

Fig. 18 - Great Western Trail, p. 7. Eggertspiele.

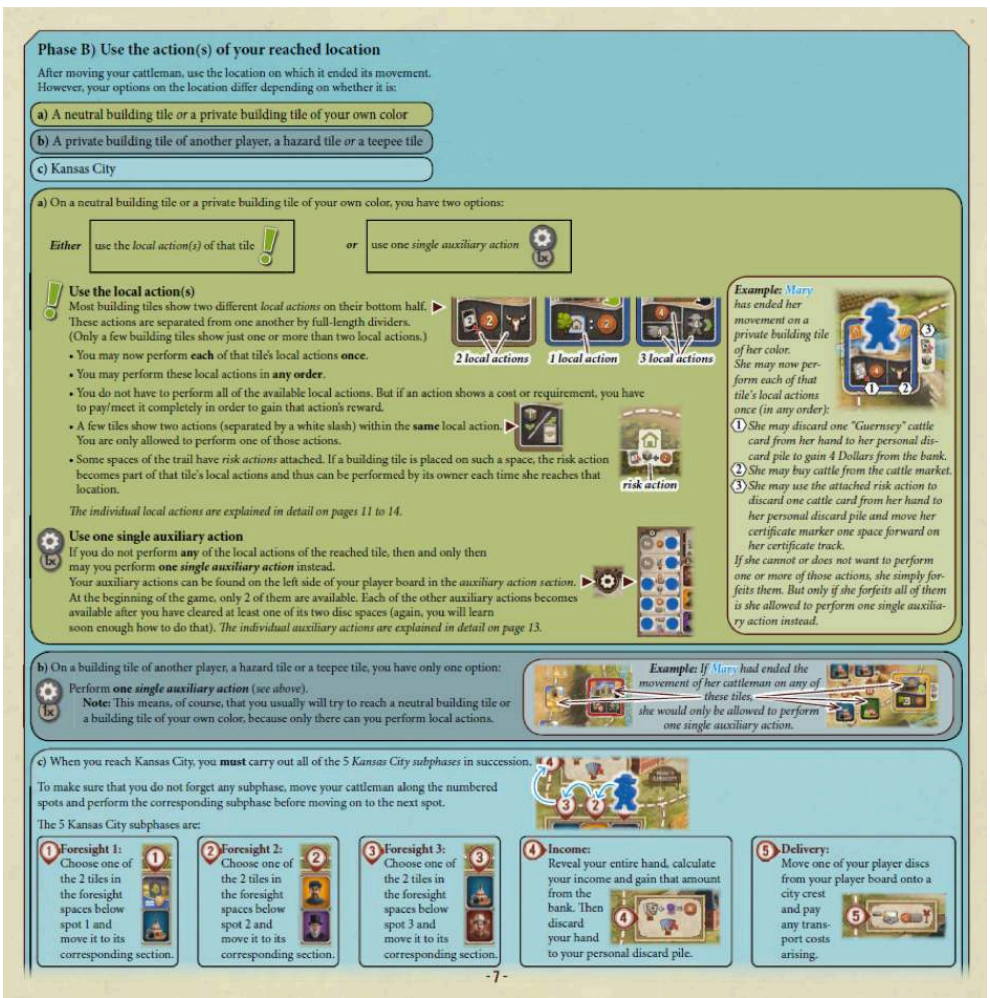



le contenu de la page : il est constitué d'un trait noir, et d'un fond bleu foncé, et ses éléments sont indexés par le titre "Phase B) [...]». Au sein de ce cadre global, des cadres indexicaux de niveau inférieur sont indiqués par des fonds colorés distincts, indexés à du texte par un système de lettres. Dans ces cadres, d'autres apparaissent, liant texte et image de toutes les façons possibles : par des flèches ou des traits, par une contiguïté entre texte en italiques et image (sous forme de légende); dans le cadre bleu ciel, situé tout en bas de la page, on trouve même un élément iconique (la petite figurine bleue) relié, par plusieurs flèches, à des chiffres successifs posés sur l'image, renvoyant eux-mêmes à diverses opérations décrites dans des cadres indexicaux de niveau inférieur, juste en dessous. On trouve le même type d'exemple de systèmes complexes à plusieurs reprises dans notre corpus (voir fig. 14 et fig. 15).

hypothèse est que ces différentes formes d'indexation servent des objectifs pédagogiques inhérents au genre notice de jeu. Il s'agit maintenant d'élaborer une typologie des fonctions didactiques qu'occupent les relations texte/image dans les règles de jeu.

\section{Fonctions didactiques}

31 Les règles ont pour rôle fondamental de construire des concepts plus ou moins complexes et/ou abstraits (étapes de jeu, personnages, achat/vente de ressources, statistiques, etc.), et de leur associer une représentation physique (un composant du jeu : une carte, un pion, un dé, un espace sur le plateau...). Ensuite, la règle doit décrire les relations entre ces représentations ${ }^{7}$ ainsi que les manipulations physiques qui y correspondent, au niveau des composants de jeu. Un bon jeu n'est néanmoins pas seulement un assemblage de mécaniques ludiques: il a aussi une forte dimension fictionnelle (appelée « thème » dans le milieu ludique) justifiant, idéalement, toutes ses mécaniques. de l'acier dans le jeu sans être connecté à une usine d'acier par un réseau de transport, alors que ce n'est pas possible pour le charbon, est justifié, dans la règle, par des considérations historiques correspondant au thème du jeu. Celui-ci se situant durant la révolution industrielle $\mathrm{du} \mathrm{XIX}^{\mathrm{e}}$ siècle: le charbon était à cette époque consommé en quantités énormes, et nécessitait un réseau de transport très efficient (rail, canaux, etc.), alors que l'acier était utilisé en très petite quantité, et pouvait donc être transporté en charrettes. Cette thématisation est souvent utilisée de la sorte, dans les notices de notre corpus, pour en favoriser l'apprentissage. Pour récapituler, les notices de jeu présentent trois aspects pédagogiques fondamentaux: (i) la "thématisation "; (ii) la construction de concepts représentés concrètement par des objets (les composants) ; (iii) la représentation des relations logiques liant ces concepts entre eux.

Concernant les rapports texte/image, trois fonctions didactiques, correspondant aux trois aspects évoqués ci-dessus, sont à l'œuvre dans les notices de jeu : la thématisation, l'exemplification, et l'explication. Ces fonctions font appel à des procédés d'indexation du texte et de l'image de plus en plus complexes, et correspondant aux trois relations que nous avons caractérisées dans la partie précédente.

Pratiques, $185-186 \mid 2020$ 


\subsection{Thématisation}

Une première catégorie d'images a vocation à construire, en association avec des textes narratifs divers, la diégèse, c'est-à-dire l'univers dans lequel le jeu de société prendra place. Ces illustrations contribuent à créer un " effet de réel », chose particulièrement prononcée dans le cadre des images légendées rentrant dans ce processus : la légende suppose effectivement une évaluation aléthique qui ne peut avoir eu lieu que dans ledit univers fictionnel, un locuteur-expert postiche s'étant chargé de la description. Mais indépendamment de l'«effet de réel » que ces illustrations et images légendées construisent, elles ont une conséquence didactique particulière dans la mesure où, en apportant un cadre d'interprétation spécifique, elles permettent de transformer ce qui n'est qu'une action banale dans la réalité - avancer un pion ou jouer une carte -, en action symbolique, dont l'interprétation fait sens au sein de l'univers diégétique. Par extension, ce contexte permet de faciliter l'apprentissage didactique en apportant une justification spécifique aux actions, notamment en termes de possibilités et de ce que le joueur peut ou ne peut pas faire (fig. 19).

Fig. 19 - Terraforming Mars, p. 5. FryxGames.

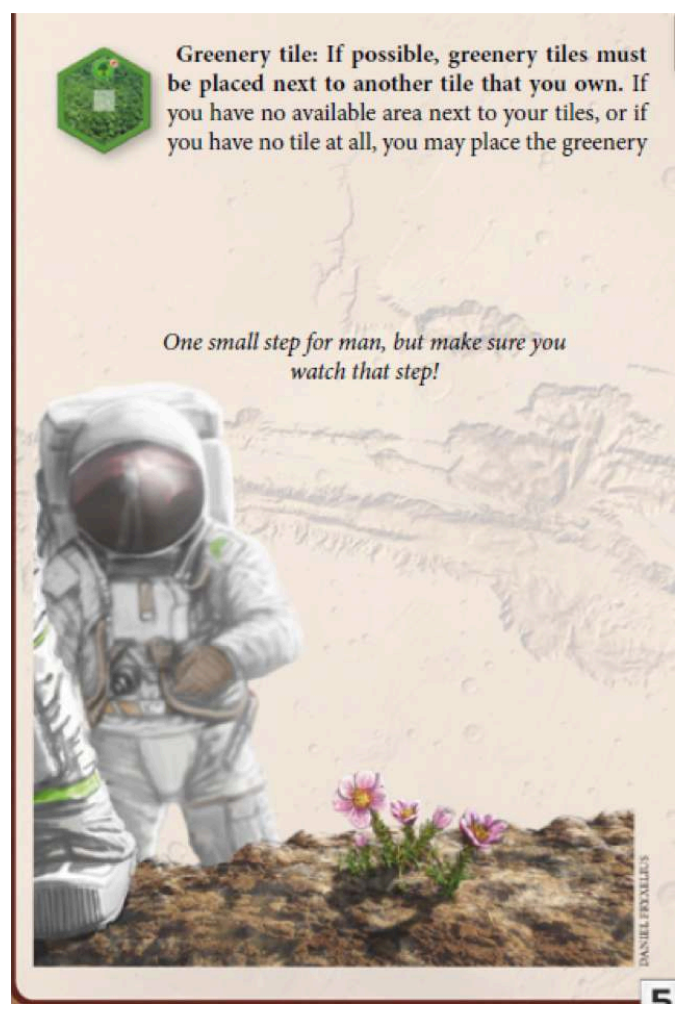

On pourra également inclure dans cette catégorie, tout ce qui est du ressort de l'habillage esthétique de la notice : fonds de page, bordures et «enluminures ». Si ces éléments ont un rôle esthétique marqué, faisant de la notice une sorte d'équivalent ludique d'un "beau livre» ou d'un livre apparat, dont le parcours doit être non seulement nécessaire mais également plaisant, ils participent également à la thématisation de l'univers mis en place. Comme nous le notions pour les images légendées entrant dans cette catégorie, ils supposent une dimension aléthique et un ensemble de valeurs qui renvoient à l'univers fictif. Ainsi en reprenant des symboles ou des codes issus directement de ces univers, la notice devient comme une extension de 
ceux-ci et fait véritablement le lien entre notre monde et le monde de la fiction en se présentant comme une sorte de codex issu de ce dernier, chose amplifiée, du reste, par les textes introductifs qui présentent généralement le jeu comme une histoire (fig. 20).

Fig. 20 - Terraforming Mars, p. 2. FryxGames.

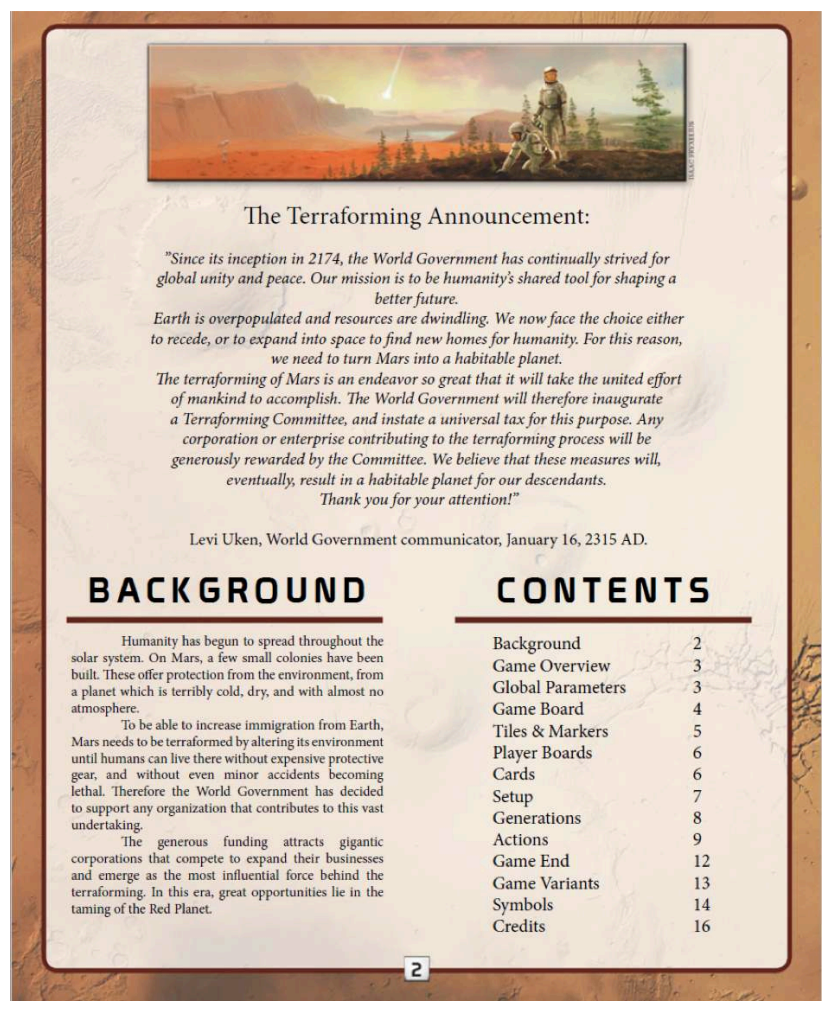

Dans ce dernier exemple, le fond rouge, imitant le sol martien, ainsi que l'illustration située en haut de la page, contribuent, avec le texte, à « fictionnaliser » l'ensemble de la notice.

\subsection{Exemplification}

Nous appelons " exemplification », la mise en lien d'un élément verbal et d'un élément graphique dans le but de permettre au joueur la reconnaissance des éléments manipulables du jeu (appelés "composants»). Nous utilisons ce terme dans une perspective extensive : l'exemplification est pour nous, ici, la caractérisation précise, à l'aide d'images, d'éléments de catégories définies par le texte. Nous distinguons cette exemplification des exemples intervenant dans les processus d'explication, que nous traiterons dans la sous-partie suivante. Avant de pouvoir jouer, il faut être capable d'identifier et de classer les composants de jeu. Les jeux de stratégie se caractérisant par une très grande variété de composants, qui ne diffèrent parfois que par un seul élément, symbole ou couleurs, et que le joueur doit apprendre à reconnaître. Cette phase d'apprentissage peut apparaître particulièrement longue pour le joueur novice. Il est possible de distinguer différents types d'interprétation selon l'appareillage texte/ image et le cadre indexical dans lequel nous les trouvons :

(i) les images suivies d'une légende, sans aucun autre type de texte. Souvent constituées en série pour faciliter une lecture totalisante, ces images ont un rôle didactique initial, lors de l'apprentissage du jeu, mais également un rôle de rappel 
et de renforcement, puisqu'elles permettent d'y revenir aisément. C'est la raison pour laquelle nous trouvons souvent un inventaire complet de ces illustrations en début ou en toute fin de notice, ces pages agissant comme une sorte d'aide-mémoire aisément accessible aux joueurs (fig. 21) ;

Fig. 21 - Star Wars Rebellion, p. 3. Fantasy Flight Games.

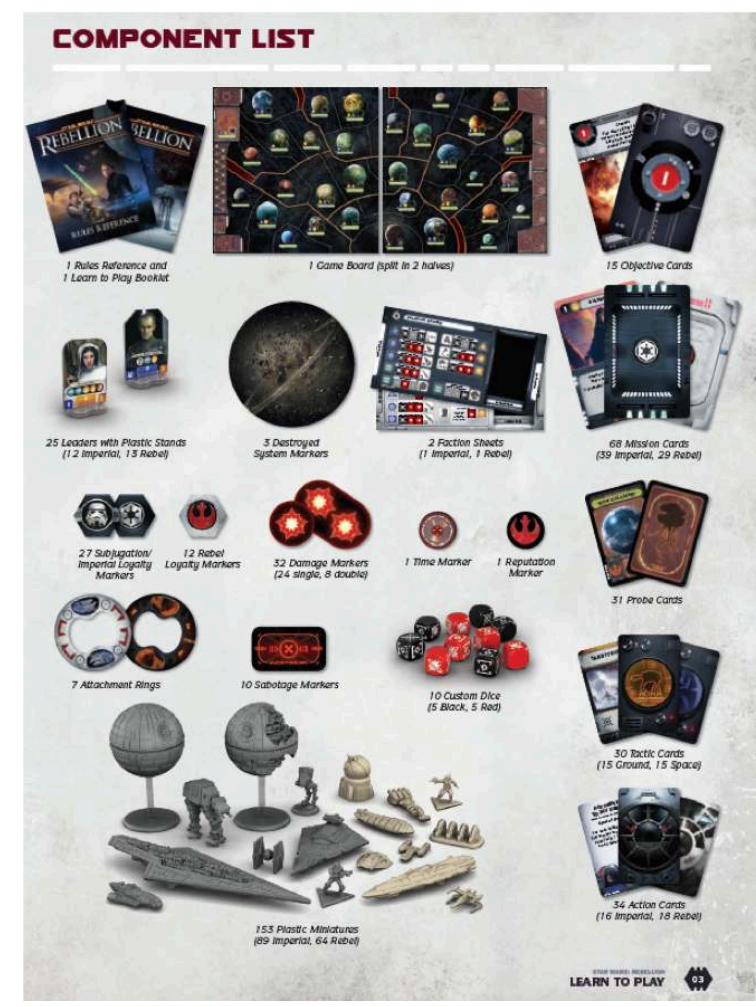

(ii) les images légendées qui suivent un texte de la notice, soit en étant disposées à la droite du texte, soit en étant situées en-dessous de celui-ci. Ces images visent à représenter par un code iconographique une description faite par le texte. Cela se rencontre notamment au commencement des notices, lors de la phase de préparation des plateaux de jeu. Comme ceux-ci doivent répondre à une grande exigence dans la position des pions, des cartes et des autres éléments, la moindre erreur ou imprécision doit être évitée. Dans la mesure où la description peut apparaître longue ou difficile à comprendre, l'illustration permet d'aider cette phase critique de la préparation du jeu, soit en précisant la position respective des éléments entre eux, soit en précisant l'objet dont il est fait mention, ou encore la manœuvre de déplacement que le joueur peut accomplir (fig. 22) ; 
Fig. 22 - Through the Ages, p. 2. Czech Games Edition.

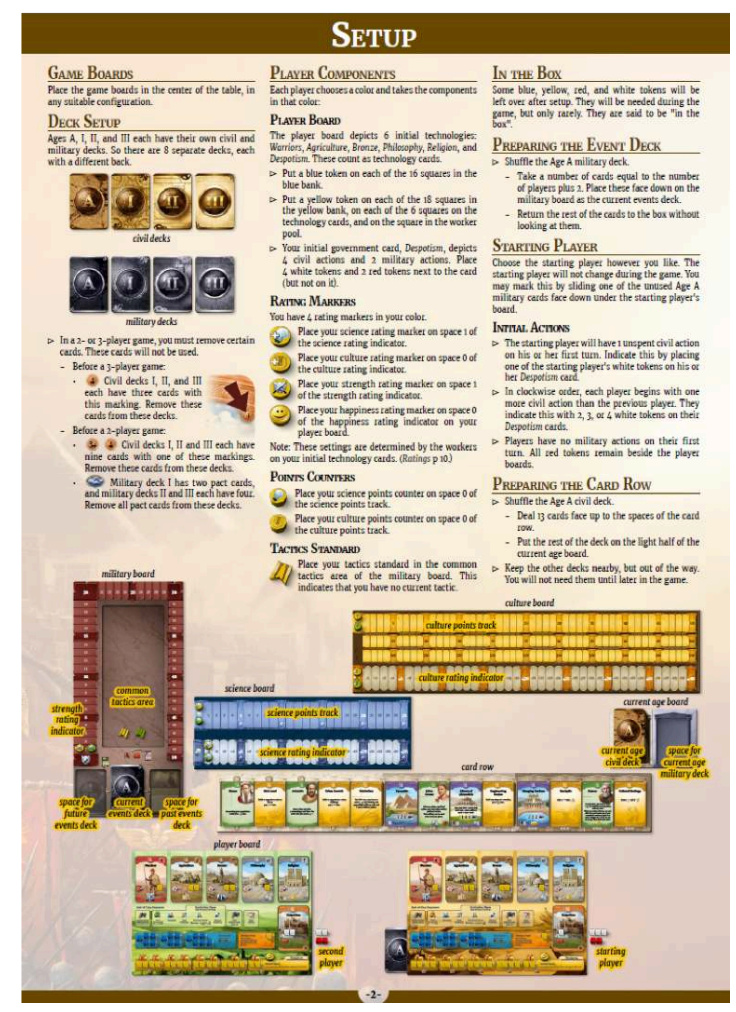

(iii) une catégorie d'image légendée semble mâtiner les deux sous-catégories précédentes : celle où la légende n'est pas en-dehors de l'illustration, mais incluse en son sein, soit par l'intermédiaire de cartouches dont les effets de couleurs, détours colorés et fond pâle, signalent leur identité extérieure à l'image, soit par l'intermédiaire d'un réseau de flèches, crochets ou autres éléments graphiques indiquant que la légende ne s'applique qu'à une portion particulière de l'image. Ces légendes ont à la fois un rôle d'apprentissage et d'aide-mémoire d'une part, mais également un rôle illustratif, en proposant une lecture séquencée d'éléments de jeu, tels les plateaux eux-mêmes, qui se composent de différentes parties signifiantes qu'il convient d'analyser et qui ne sont donc point monosémiques à l'instar, par exemple, des jetons de jeu. Nous retrouvons d'ailleurs cette même idée dans le cadre d'une indexation plus forte entre le texte et l'image, où la lecture de l'image est séquencée par une série de puces numérotées renvoyant à un texte explicatif à ses côtés, que nous décrirons ci-après (fig. 23). 
Fig. 23 - Gaia Project, p. 5. Z-Man Games, Inc.

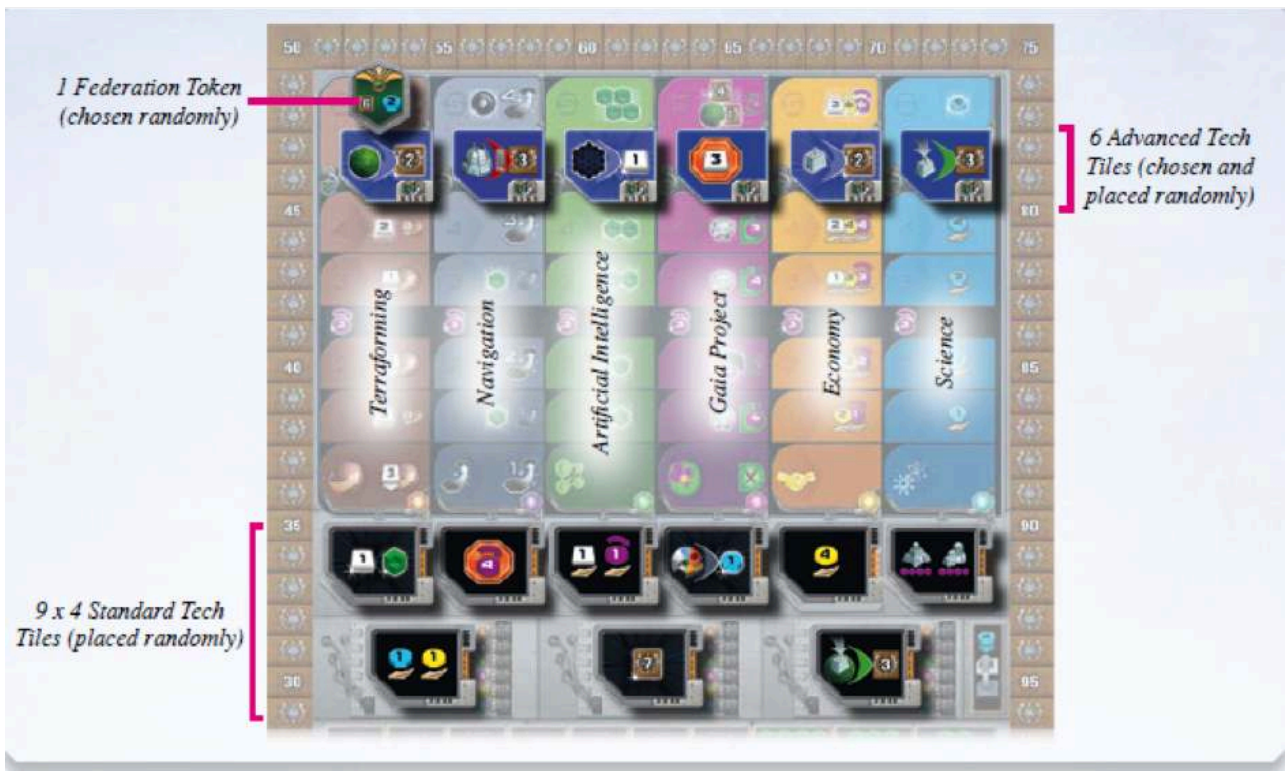

37 Quelle que soit la sous-catégorie de légendes cependant, le principe demeure identique : relier une image à un élément textuel par la création d'un cadre indexical, l'association se faisant parfois par un «sur-marquage " de leurs correspondances sémantiques, par un trait, une flèche, une accolade ou autre procédé similaire. Les objets représentés par ce type d'images sont effectivement, soit uniques dans le cadre $\mathrm{du}$ jeu, le plateau, ou alors certains pions ou cartes uniques en leur genre par exemple, soit présentés comme parangons d'une série d'objets similaires, qui rempliront les mêmes rôles au sein de la partie. C'est ce que nous avons notamment dans les "pages récapitulatives » que nous évoquions précédemment, où l'inventaire ne saurait être exhaustif lorsqu'il y a plus de cinq cents cartes d'une certaine catégorie ou, même, une dizaine de jetons d'une autre. (fig. 24). 
Fig. 24 - Gloomhaven, p. 2. Cephalofair Games.

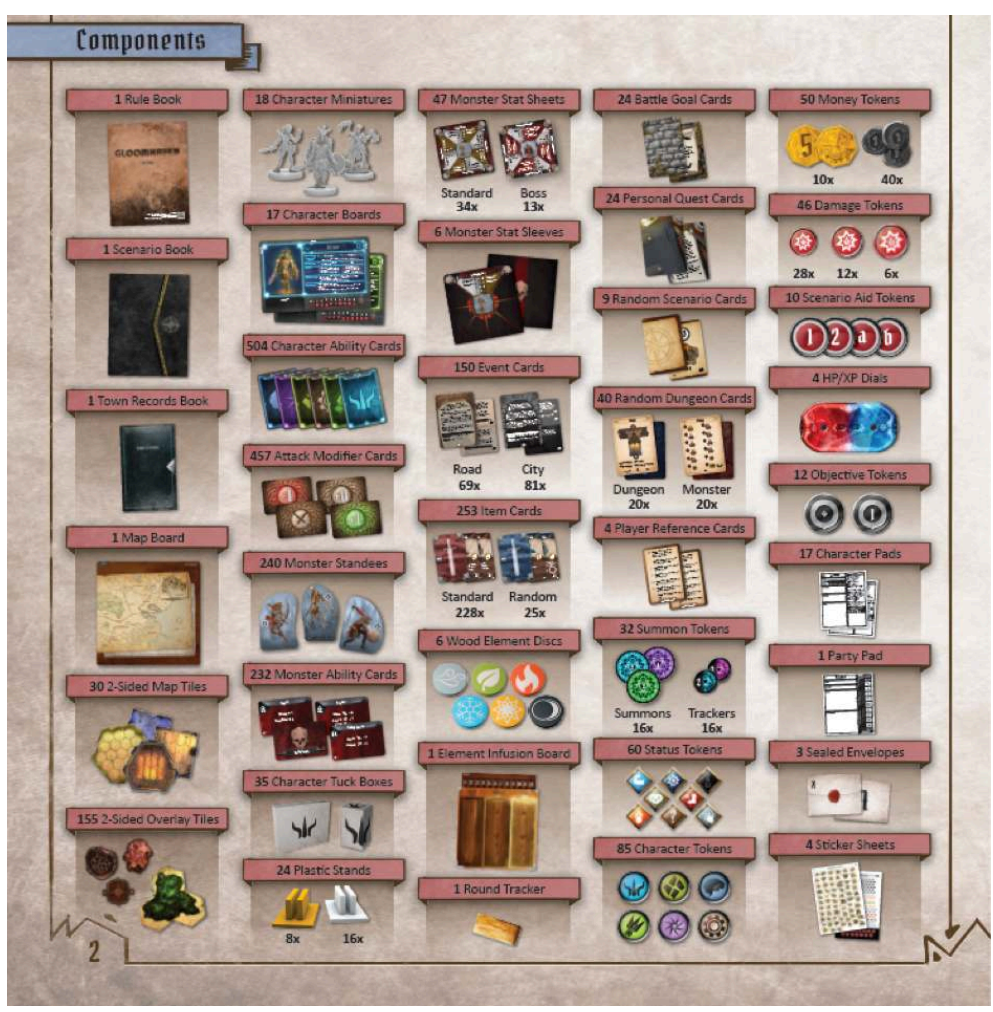

Partant, dans ce dernier cas de figure, la légende n'a pas qu'une seule valeur descriptive dans ce dernier cas de figure, mais également une portée catégorisante, la chose étant généralement indiquée par le fait que l'image légendée est: soit (i) une collection d'images de la même catégorie, soit (ii) complétée par une indication numérique indiquant le nombre d'objets auxquels s'applique la légende. Ces légendes permettent de créer des «modèles mentaux » facilitant la compréhension et la mémorisation du rôle de ces objets, selon des mécanismes qui ont été décrits en psychologie cognitive, par exemple par A. M. Glenberg \& W. E. Langston (1992).

39 Nous sommes ici dans la logique de l'hyperonymie ou du prototype ${ }^{8}$, et la valeur de la légende vaut donc autant pour sa portée descriptive, (elle associe à un objet concret une valeur abstraite et symbolique, tel pion représentant tel personnage ou telle carte telle possibilité d'action), que pour sa portée classifiante (elle permet de construire des séries d'objets similaires entre eux). Notons que cette classification pouvait s'effectuer d'ores et déjà par un rapprochement d'objets identiques entre eux, mais leur regroupement au sein d'une même légende amplifie cette reconnaissance et participe pleinement de l'apprentissage du "savoir-jouer ». Les légendes effectuent ainsi une passerelle entre un univers concret et un univers abstrait par l'intermédiaire de cette phase didactique de reconnaissance des objets à manipuler ou avec lesquels interagir, quand bien même elles ne seraient pas l'objet d'une explication approfondie quant aux rôles ou prérogatives de l'objet en question.

\subsection{Explication}

La troisième fonction didactique des relations texte/image du corpus est d'expliquer comment les éléments de jeu, associés à des concepts et caractérisés grâce à la fonction 
d'exemplification décrite ci-dessus, s'associent les uns aux autres pour fabriquer des mécaniques ou des mécanismes ludiques. Nous entendons par "mécanisme ludique " un processus logique et narratif conduisant d'une situation initiale, caractérisée par un positionnement particulier des éléments de jeu, à une situation finale différente de la situation initiale. Entre les deux, des règles logiques (constituées d'opérateurs classiques de la logique, du type "si X, donc $\mathrm{Y}$ ", ou d'opérations mathématiques plus ou moins complexes) permettent de conduire le processus de transformation conduisant de la situation initiale à la situation finale. Ainsi, par exemple, l'achat ou la vente de ressources constituent des mécanismes ludiques, physiquement incarnés par les composants du jeu : le joueur ajoute des composants à sa réserve, ou en enlève, etc. Les mécanismes ludiques peuvent être de complexité variable, et peuvent se combiner pour élaborer une «mécanique ». Par exemple, dans Brass: Birmingham, comme dans de nombreux jeux de stratégie économiques, la présence d'un marché est une mécanique mettant en jeu, entre autres, des mécanismes d'achat et de vente de ressources.

41 Rien d'étonnant, dans ce cas de figure, à ce que les combinaisons texte/image s'organisent de façon séquentielle et/ou hiérarchique pour expliquer ces mécanismes, et ce à plusieurs niveaux. En effet, pour expliquer un mécanisme, il faut en décrire les étapes successives, logiques, menant de la situation initiale à la situation finale, ce qui se manifeste souvent dans le corpus par l'emploi conjoint de flèches, de chiffres et de lettres (fig. 25).

Fig. 25 - Brass : Birmingham, p. 9. Roxley.

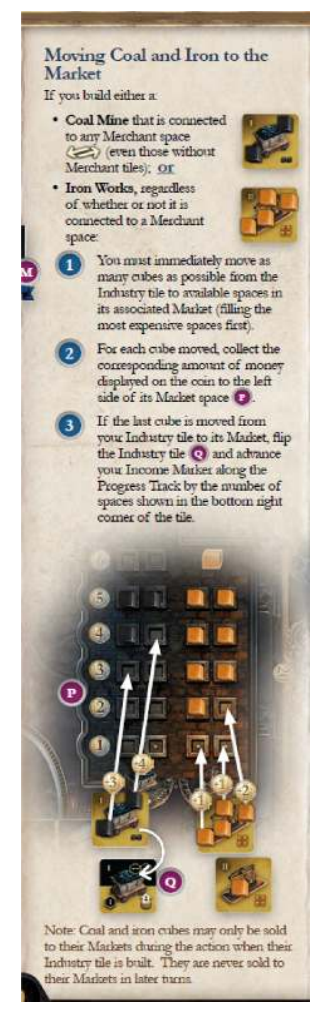

On décrit ici l'un des mécanismes permettant de faire fonctionner le marché, dans Brass: Birmingham, soit le mouvement du charbon et de l'acier, représentés par des cubes noirs et orange. Les images et le texte interagissent à plusieurs niveaux. D'abord, tout en haut de l'exemple, deux images correspondent à la fonction d'exemplification 
extensive, permettant de faire le lien entre ce qui est appelé "Coal Mine » et «Iron Works » et les composants qui les représentent. Cette exemplification est ensuite mise au service d'une explication des différents mécanismes permettant de faire fonctionner le marché, ici la séquence numérotée de 1 à 3 , et qu'il faut suivre. À chaque fois, un bloc de texte correspond à une étape ; ces blocs de texte sont composés de discours injonctifs, à l'impératif ou, en tout cas, présentant une forte modalité injonctive. Au sein du texte, des lettres, $\mathrm{P}$ et $\mathrm{Q}$, renvoient à des zones de l'image du dessous. Il s'agit, pour la lettre « $\mathrm{P}$ », d'exemplification : les mots qui précèdent $\mathrm{P}$ (Market space, « espace de marché ») renvoient à un concept, qui est indexé à une zone visible du plateau de jeu, représentée par l'image. Les mots qui précèdent la lettre " $Q$ », par contre, renvoient à une flèche et décrivent un mécanisme : flip the Industry tile (« retournez la tuile industrie »), ce qui est représenté au niveau de l'image par une flèche courbe qui indique qu'il faut retourner l'élément de jeu. Enfin, les flèches droites relient, dans l'image, les différents éléments indexés précédemment lors de la phase d'exemplification. Sur ces flèches, des éléments de jeu sont représentés, la monnaie, sur laquelle des représentations graphiques $(+1,+1,+2$, etc.) indiquent la quantité d'argent que reçoit le joueur en déplaçant des éléments de jeu d'un endroit à un autre, modifiant la situation initiale pour parvenir à une situation finale. Ces flèches, en relation avec les chiffres qui leur sont surimposés relèvent bien, quant à elles, de l'explication du mécanisme et non de la simple exemplification : il s'agit de montrer que le déplacement de certains éléments (les cubes de charbon et d'acier du jeton industrie vers le marché) entraîne le déplacement d'autres éléments (les jetons de monnaie de la banque vers la réserve du joueur).

La séquence de texte, numérotée de 1 à 3 , et les images, fonctionnent, à un niveau supérieur, comme un seul bloc dont les constituants sont interdépendants (ce qui est d'ailleurs marqué par la présence d'un cadre indexical regroupant tous ces éléments). L'ensemble a une seule fonction : expliquer un des mécanismes sous-tendant la mécanique du marché. Cette fonction d'explication est la plus complexe des fonctions pédagogiques des rapports texte/image car elle met en jeu, de façon séquentielle et hiérarchique, toutes les possibilités d'indexation entre le texte et l'image, ainsi que les autres fonctions pédagogiques comme l'exemplification et, parfois, la thématisation ; de plus, elle joue à plusieurs niveaux, du micro (au niveau des flèches droites de notre exemple, qui relèvent d'une explication très localisée) au macro (au niveau du cadre indexical global, intitulé " Moving coal and iron to the market »).

\section{Séquentialité des fonctions didactiques}

Nous faisons ici l'hypothèse que les notices sont aussi organisées, au niveau macrostructurel, en séquences bien précises, qui favorisent l'apprentissage des règles du jeu. Notre propos est ici de repérer ces séquences, et d'observer si des tendances quantitatives, en termes de fonctions pédagogiques des relations texte/image, leur correspondent. Nous avons compté les exemples sans assistance automatisée, en nous fondant sur notre interprétation, aussi faut-il prendre les chiffres avancés avec prudence : en effet, comme nous l'avons vu, s'il est assez facile d'identifier la fonction d'exemplification, la fonction d'explication, elle, est bien plus complexe à caractériser car elle fait parfois appel aux deux autres fonctions répertoriées. Nous avons donc 
compté certaines occurrences deux fois, une combinaison explicative reposant souvent sur un ou plusieurs cas d'exemplification.

\subsection{Séquences didactiques dans les notices du corpus}

Fig. 26 - Gaia Project, p. 2. Z-Man Games, Inc.

\section{TABIF OF BDIIIIITS}

Components
Game Setup
Goal and Gameplay
The Power Gycle
The $\mathbf{6}$ Rounds of Play
I. Income
II. Gaia
III. Actions
1) Build a Mine
2) Start a Gaia Project
3) Upgrade Existing Structure
4) Form a Federation
5) Research Progress

5) Research Progress

\author{
6) Power and QI.C. Actions \\ 7) Special Actions \\ 8) Pass \\ 9) Free Actions \\ 10) Passive Action: Charging Power
}

IV. Clean-Up

\section{Game End and Scoring}

Advanced Setup: Beyond the First Game

Appendices

I. Factions

II. Research Board/III. Action Spaces

IV. Round Boosters / V. Round Scoring Tiles

VI. Tech Tiles

Fig. 27 - Twilight Struggle, p. 1. GMT Games.

\section{TAB LE OF CONTENTS}

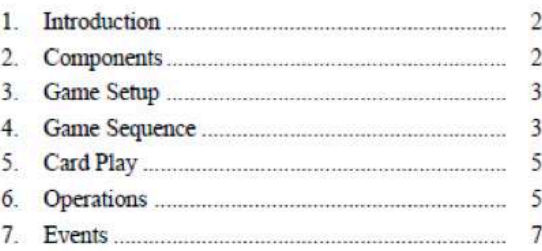

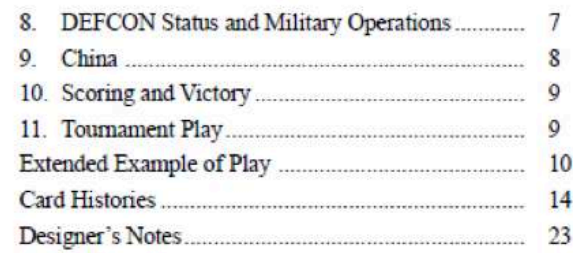

Dans ces deux sommaires, issus respectivement de Gaia Project et de Twilight Struggle, nous retrouvons bien :

1. la présentation des composants (Components);

2. la mise en place du plateau (Game Setup) ;

3. la description des mécanismes du jeu, qui composent l'essentiel de ces notices (de Goal and Gameplay, p. 8, à Clean-Up, p. 17 pour Gaia Project, de Game Sequence, p. 3 à China, p.8 pour Twilight Struggle) ;

4. le calcul du score (Game end and scoring/scoring and victory).

Les chapitres terminaux sont quant à eux consacrés à des modes de jeu alternatifs et à différents appendices.

47 Ces séquences suivent l'ordre chronologique d'une partie, ce qui a une fonction didactique à deux niveaux: d'abord, parce que cela permet tout simplement de se représenter une partie, avant sa conduite effective; ensuite, parce que cela favorise aussi l'explication des règles aux autres joueurs. En effet, il est rare que tous les joueurs débutants fassent l'effort de lire les règles; la plupart du temps, un seul le fait, qui les explique ensuite aux autres. 


\subsection{Séquentialité des fonctions didactiques} didactiques (composants/mise en place/mécanismes/calcul du score), et que nous faisons le décompte du type de relations texte/image que nous avons distingués, nous obtenons les résultats suivants :

\begin{tabular}{|l|l|}
\hline Moment didactique & Type de relation texte/image \\
\hline Composants & $\begin{array}{l}\text { Thématisation : } 7 \\
\text { Exemplification : } 212 \\
\text { Explication : } 186\end{array}$ \\
\hline Mise en place & $\begin{array}{l}\text { Thématisation }: 4 \\
\text { Exemplification }: 145 \\
\text { Explication }: 147\end{array}$ \\
\hline Mécanismes & $\begin{array}{l}\text { Thématisation : } 9 \\
\text { Exemplification : } 196 \\
\text { Explication }: 333\end{array}$ \\
\hline Calcul \\
du score
\end{tabular}

Ainsi retrouvons-nous une majorité d'exemples lors de la phase de présentation des composants, ce qui rejoint nos commentaires précédents sur cette phase de la notice. Les phases présentant les mécanismes et le calcul du score, demandant de mettre en relation des éléments complexes entre eux, sont surtout illustrées par des processus d'explication. La mise en place de la partie, en revanche, mâtine ces deux types de fonctions indexicales pour des raisons, une fois encore, détaillées précédemment. Cependant, on notera que cette phase permet de faire le lien entre la phase de présentation des composants et l'explication des mécanismes du jeu. Nous pouvons postuler que cela facilite l'introduction des codes qu'utilisera la notice lors de la présentation du cœur de la partie, en opérant une transition entre une phase surtout marquée par l'exemple et une autre, surtout identifiée par l'explication.

Tous ces éléments peuvent être mis en relation avec le rôle de la notice, qui doit davantage expliquer qu'illustrer, le rôle mémoriel qu'offrent les légendes et les exemples étant, in fine, secondaire aux côtés de l'explication des mécanismes de jeu : autant le joueur peut rapidement se référer à l'inventaire des cartes et des jetons pour guider sa partie, autant il convient d'avoir une idée précise de ses modalités d'action pour avoir une pratique de jeu efficace. Les exemples ont ainsi davantage un rôle de soutien que de progression didactique, l'apprentissage se faisant quasi exclusivement par les relations texte/image de type explicatif.

On rappellera également que ces notices suivent une progression globalement chronologique, les explications accompagnant cette idée. Les premiers moments de la notice sont généralement dédiés à l'établissement du cadre fictionnel, ce qui justifie la présence d'illustrations ; nous avons ensuite l'inventaire des objets du jeu, accompagné par des exemples ; puis les explications à proprement parler, qui accompagnent les moments clés de la partie, la préparation du plateau de jeu, le déroulement de la partie 
puis le calcul des points de victoire. On aura occasionnellement, en général à la fin de la notice, des précisions concernant des règles additionnelles, ou des détails venant compliquer la conduite de la partie, mais ces éléments ne mettent pas en péril ce canevas chronologique.

La progression chronologique de l'explication, au regard d'une progression davantage diégétique, dessine les contours d'une logique didactique fonctionnant en acte plutôt qu'en dire. Cette logique conduit les joueurs à progresser dans leur apprentissage en suivant le déroulement temporel d'une partie spécifique; chaque explication vient alors justifier, exemplifier ou schématiser une action ou un problème rencontré à ce moment-là de la progression du jeu. Cela, en association avec la comparaison que le joueur fera entre la notice et le plateau physique qui se trouve effectivement devant lui, facilite notablement l'apprentissage. Dans ces notices, l'explication n'est pas un préalable à l'apprentissage, mais un accompagnement, ce qui justifie en retour non seulement la très forte présence des relations de type explicatif dans les notices, mais également leur fréquence puisque nous les retrouvons - en nombre - à tous les moments de leur progression.

\section{Conclusion}

53 Le parcours des notices du corpus a permis de mettre au jour différents types de rapports scripto-iconiques, en relation avec plusieurs stratégies didactiques. Malgré la disparité des différents jeux étudiés et leur complexité relative, des patrons émergent quant au modèle et au genre notice de jeu. Ces patrons, se fondant sur des cadres indexicaux diversement constitués, accompagnent la chronologie de la partie et sont introduits progressivement, afin de faciliter la phase d'apprentissage. Cependant, la chronologie est modifiée par l'intermédiaire d'entrées thématiques et de pages récapitulatives, ce qui permet à la notice d'être un outil de consultation précieux quel que soit le niveau d'apprentissage du joueur, débutant, intermédiaire voire confirmé, en général après une première appropriation de la règle.

Ainsi les stratégies mises en place dans les relations texte/image proposent-elles une progression intelligente de la dynamique didactique : si l'explication, attendue dans le genre général de la notice de jeu, domine le corpus, elle se mélange à l'exemplification, par l'intermédiaire de légendes, pour construire un parcours mesuré allant de la mise en place d'un univers fictionnel au calcul des points de victoire, en passant par une phase de présentation des composants du jeu, jetons et plateaux, et de ses mécanismes. En alternant la fréquence des différents types de rapport, qui se caractérisent par la mise en place de cadres indexicaux de plus en plus complexes, et en proposant une transition entre exemples et explications grâce à une phase intermédiaire de mise en place des éléments de jeu, les notices de jeu facilitent l'apprentissage des codes employés et tiennent aussi compte des pratiques des joueurs : les règles sont, certes, faites pour être lues, mais aussi pour être expliquées à d'autres, et consultées lors de points de litiges. L'impact de ces pratiques sur les modalités de production et de réception des notices de jeux devrait être étudié.

Les notices de jeu diffèrent donc notablement des autres types de discours programmateurs, ou des discours d'incitation à l'action (Adam, 2001). Elles présentent à la fois un cadre chronologique accompagnant la partie, à l'image d'une recette de cuisine qui suivrait la préparation décrite, mais également une séquentialité marquée 
de sa progression, en isolant différents moments d'importance signifiés par différentes explications, à l'instar des notices de médicaments. Les notices de jeu proposent un apprentissage que l'on pourrait appeler «chrono-thématique », d'une grande plasticité et convenant autant à des joueurs débutants qu'à des joueurs d'un niveau avancé ; et qu'il y ait, ou non, concomitance entre actions et déroulement temporel de la partie, la séquentialité proposée par ces notices facilite fortement les apprentissages en introduisant des entrées thématiques plus ou moins libres au sein d'un déroulement chronologique plus fortement chevillé à la pratique concrète du jeu.

La notice de jeu doit donc être considérée comme un ensemble totalisant. L'interprétation difficile des points de détail se résout lors de la prise en compte de l'intégralité du document, qui œuvre à un apprentissage clair malgré la complexité de certains jeux de stratégie.

\section{BIBLIOGRAPHIE}

ADAM, J.-M. (2001). « Types de textes ou genres de discours ? Comment classer les textes qui disent de et comment faire? ». Langages 141. Paris : A. Colin. p. 10-27. En ligne : https://www.persee.fr/ doc/lgge_0458-726x_2001_num_35_141_872.

BENVENISTE, É. (1947). « Le jeu comme structure ». Deucalion. Cahiers de philosophie 2, p. 161-167.

GLENBERG, A. M. \& LANGSTON, W. E. (1992). « Comprehension of Ilustrated Text: Pictures Help to Build Mental Models ». Journal of Memory and Language 31 (2). p. 129-151. Amsterdam : Elsevier.

HALTÉ, P. (2017). « Positionnement syntaxique des interjections et des émoticônes : modalisation, portée, visée ». Les Cahiers de praxématique 69. Montpellier : Presses universitaires de la Méditerranée. En ligne : https://journals.openedition.org/praxematique/4680.

HÉNAULT, A. (2008). « Image et texte au regard de la sémiotique ». Le Français aujourd'hui 161.

Paris : A. Colin., p. 11-20. En ligne : https://www.cairn.info/revue-le-francais-aujourd-hui-2008-2page-11.htm.

KLEIBER, G. (1990). La sémantique du prototype. Catégories et sens lexical. Paris : Presses universitaires de France.

KLINKENBERG, J.-M. (2008). « La relation texte-image. Essai de grammaire générale ». Bulletin de la Classe des Lettres et des sciences morales et politiques 19, p. 21-79. En ligne : https://www.persee.fr/ doc/barb_0001-4133_2008_num_19_1_23906.

MACEDO-ROUET, M. \& ROUET, J.-F. (2011). « Documents multiples : processus et difficultés de lecture ». Argos 48. 


\section{Ludographie}

BELTRAMI, D. et al. (2017). Twilight Imperium (Fourth Edition). Learn to play. Rosseville : Fantasy Flight Games. En ligne : https://images-cdn.fantasyflightgames.com/filer_public/f3/c6/ f3c66512-8e19-4f30-a0d4-d7d75701fd37/ti-k0289_learn_to_playcompressed.pdf. BROWN, G. et al. (2018). Brass: Birmingham rulebook. Calgary : Roxley. En ligne : https://roxley.com/ products/brass-birmingham-deluxe-edition?currency=EUR\#downloads. CHILDRES, I. (2017). Gloomhaven: Final Rules - Low Resolution. Cephalofair Games. En ligne : https:// boardgamegeek.com/filepage/136993/gloomhaven-final-rules-low-resolution.

CHVÁTIL, V. (2015). Through the Ages: A New Story of Civilization. Kladno : Czech Games Edition. En ligne : https://czechgames.com/files/rules/through-the-ages-new-story-rules-en.pdf.

DAVIAU, R. \& LEACOCK, M. (2015). Pandemic Legacy Season 1 rulebook. Rosseville : Z-Man Games, Inc. En ligne : https://www.zmangames.com/en/products/pandemic-legacy-season-1/.

DRÖGEMÜLLER, J. \& OSTERTAG, H. (2017). Gaia Project. Rosseville : Z-Man Games, Inc. En ligne : https:// images.zmangames.com/filer_public/ce/89/ce890bfd-227e-4249-a52a-976bc5f20d19/

en_gaia_rulebook_lo.pdf.

FRYXELIUS, J. (2016). Terraforming Mars. Vellinge : FryxGames. En ligne : https:// www.fryxgames.se/TerraformingMars/TMRULESFINAL.pdf.

GUPTA, A. \& MATTHEWS, J. (2005). Twilight Struggle. Hanford : GMT Games. En ligne : https:// www.gmtgames.com/p-775-twilight-struggle-deluxe-edition-7th-printing.aspx.

KONIECZKA, C. (2016). Star Wars: Rebellion. Rosseville : Fantasy Flight Games. En ligne : https:// www.fantasyflightgames.com/en/products/star-wars-rebellion/.

PFISTER, A. (2016). Great Western Trail. Hambourg : Eggertspiele. En ligne : https://

boardgamegeek.com/filepage/137068/great-western-trail-english-rule-book.

\section{NOTES}

1. Tous les manuels cités sont disponibles gratuitement et publiquement sur les sites des éditeurs ou sur https://boardgamegeek.com.

2. Par "fonction didactique », nous entendons le(s) rôle(s) que jouent les procédés d'association texte/image dans l'apprentissage d'un «savoir-jouer». Ces fonctions didactiques s'appuient sur des fonctions pragmatiques (performativité implicite des consignes, actes de langage injonctifs, "pointage » impératif par l'intermédiaire de flèches, etc.), marquées dans des systèmes sémiotiques différents (verbal et visuel). Nous n'évoquerons ici que très peu ces fonctions pragmatiques, qui devraient faire l'objet d'une étude à venir.

3. Ces jeux sont présentés dans la «ludographie» disponible dans la partie «bibliographie » en fin d'article.

4. En ligne : https://boardgamegeek.com.

5. Nous entendons ici par "énoncé » le produit d'une énonciation (avec sa deixis propre), quel que soit le système sémiotique (verbal, visuel, gestuel, etc.) qui la constitue. Nous opposons énoncé verbal et visuel en suivant J.-M. Klinkenberg (2008) ; 
nous préférons, comme lui, "visuel » à "iconique », car l'iconicité - en sémiotique peircienne notamment - désigne simplement une relation de ressemblance entre un signe et un objet, et n'est donc pas cantonnée au visuel (il existe des énoncés verbaux iconiques, comme, par exemple, les onomatopées).

6. Pour plus d'informations sur ces emplois particuliers, voir les travaux sur l'intégration des émoticônes et des émojis à la chaîne syntagmatique, qui relève de la même problématique (Halté, 2017).

7. Ces relations, associées en divers ensembles, constituent ce qu'on appelle les "mécaniques» de jeu.

8. On se reportera notamment à G. Kleiber (1990, p. 21-117) pour une analyse de la « sémantique du prototype ».

\section{RÉSUMÉS}

Cet article explore les relations texte-image dans un corpus de notices de jeu de stratégie, sous l'angle de leurs fonctions didactiques. Notre hypothèse est que ces notices, dont l'objectif explicite est de permettre l'apprentissage de règles de jeu, sont le plus souvent constituées de textes et d'images dont les relations correspondent à différents moments didactiques. Nous dressons dans un premier temps, un inventaire des relations texte-image du corpus, en nous fondant sur la notion de « cadre indexical» (Klinkenberg, 2008). Ensuite, nous montrons que ces différentes relations servent des fonctions didactiques plus ou moins spécifiques: l'exemplification, la thématisation, et l'explication. Enfin, nous étudions l'aspect séquentiel des fonctions didactiques dans les notices de jeux de stratégie, c'est-à-dire la succession «chronologique » de moments didactiques, portés par des relations texte-image variées.

This study is about text/image relations in strategy game manuals. We analyze those relationships from a didactical point of view. Our hypothesis is that strategy game manuals are constituted of texts and images that interact in different ways, which are relevant to different didactical moments. To begin with, we use the concept of "deictic frame" (Klinkenberg, 2008) to categorize the different types of text/image relations. Then we show that these relations are used in precise didactical functions: exemplification, thematization, and explanation. Finally, we study the didactical functions from a sequential standpoint: we show that different text/image relations appear according to a chronological succession of didactical moments.

\section{INDEX}

Keywords : text/image relations, semiotics, didactics, game manuals.

Mots-clés : relation texte/image, sémiotique, didactique, notices de jeu. 


\section{AUTEURS}

\section{MATHIEU GOUX}

Université de Caen Normandie, Crisco, F-14032 Caen, France

\section{PIERRE HALTÉ}

Université de Paris-Descartes, EDA, F-75006 Paris, France 